\title{
Liver fatty acid-binding protein (L-FABP) promotes cellular angiogenesis and migration in hepatocellular carcinoma
}

\author{
Chung-Yu Ku ${ }^{1}$, Yu-Huei Liu ${ }^{2}$, Hsuan-Yuan Lin ${ }^{3}$, Shao-Chun Lu' ${ }^{1}$, Jung-Yaw Lin ${ }^{1,3}$ \\ ${ }^{1}$ Institute of Biochemistry and Molecular Biology, College of Medicine, National Taiwan University, Taipei City, Taiwan \\ ${ }^{2}$ Graduate Institute of Integrated Medicine, China Medical University, Taichung City, Taiwan \\ ${ }^{3}$ Department of Life Science, National Taiwan Normal University, Taipei City, Taiwan \\ Correspondence to: Shao-Chun Lu, e-mail: Isc@ntu.edu.tw \\ Jung-Yaw Lin, e-mail: linjy@ntu.edu.tw
}

Keywords: angiogenesis, hepatocellular carcinoma, liver fatty acid-binding protein, vascular endothelial growth factor Received: July 22, $2015 \quad$ Accepted: January 29, 2016

Published: February 22, 2016

\section{ABSTRACT}

Liver fatty acid-binding protein (L-FABP) is abundant in hepatocytes and known to be involved in lipid metabolism. Overexpression of L-FABP has been reported in various cancers; however, its role in hepatocellular carcinoma (HCC) remains unclear. In this study, we investigated L-FABP and its association with vascular endothelial growth factors (VEGFs) in 90 HCC patients. We found that L-FABP was highly expressed in their HCC tissues, and that this expression was positively correlated with that of VEGF-A. Additionally, L-FABP significantly promoted tumor growth and metastasis in a xenograft mouse model. We also assessed the mechanisms of L-FABP activity in tumorigenesis; L-FABP was found to associate with VEGFR2 on membrane rafts and subsequently activate the Akt/mTOR/P70S6K/4EBP1 and Src/FAK/cdc42 pathways, which resulted in up-regulation of VEGF-A accompanied by an increase in both angiogenic potential and migration activity. Our results thus suggest that L-FABP could be a potential target for HCC chemotherapy.

\section{INTRODUCTION}

Hepatocellular carcinoma (HCC), the most common type of liver cancer, is notoriously resistant to systemic therapies and has a relatively high recurrence rate. The poor prognosis associated with HCC causes more than 700,000 deaths annually and has become the third leading cause of cancer-related death worldwide $[1,2]$. Angiogenesis plays an important role in the progression and metastasis of $\mathrm{HCC}$, and vascular endothelial growth factors (VEGFs) are critical drivers of the "angiogenic switch" in tumors, which is a process that stimulates the formation of new blood vessels to supply the nutrients and oxygen required for sustained tumor growth [1]. VEGF ligands bind to three similar receptor tyrosine kinases, namely VEGFR1 (FLT1), VEGFR2 (KDR), and VEGFR3 (FLT4), by different affinities; however, VEGFR2 is the major receptor for VEGF-induced signaling and therefore serves as a major therapeutic target [3]. Because HCC is often diagnosed at an advanced stage and is accompanied by tumor angiogenesis and metastasis, VEGF-targeted therapies may have therapeutic benefits $[2,4]$.

Liver fatty acid-binding protein (L-FABP), a member of the FABP family, is expressed abundantly in the cytoplasm and can bind hydrophobic lipid ligands with a high specificity. L-FABP uniquely binds two ligand molecules (long chain fatty acids) or various hydrophobic molecules (e.g., cholesterol and bile acids) [5]. Furthermore, L-FABP interacts with the plasma membrane to enhance cholesterol transfer or participate in membrane microdomain alteration [6]; however, the mechanisms underlying L-FABP activity are currently unclear.

Overexpression of L-FABP has been observed in various cancers, including liver, lung, gastric, and colon cancers. Moreover, several studies have indicated that L-FABP expression is correlated with VEGF expression in HCC $[7,8]$. The precise mechanisms underlying this correlation remain unknown; therefore, in this study, we investigated the association between L-FABP and VEGF in $90 \mathrm{HCC}$ patients. We found that L-FABP was highly expressed in the tumor tissue of these patients compared 
with expression in their normal adjacent tissue, and we observed a positive correlation between L-FABP and VEGF-A expression. Overall, our study suggests that L-FABP participates in HCC malignancy and could serve as a potential target for HCC therapy.

\section{RESULTS}

\section{Overexpression between L-FABP and VEGF-A in HCC tissues is positively correlated}

Following immunohistochemical staining, expression levels of L-FABP in 90 pairs of tissue (HCC tumor and normal adjacent tissue) were classified by staining intensity as negative, weak, moderate, or strong; the associated photographs are shown in Figure $1 \mathrm{~A}$. Expression of L-FABP was significantly higher in all tumor tissues (HCC with or without cirrhosis) compared with NAT (Table 1, $p=0.012$ ). In addition, the expression of L-FABP was positively correlated with that of VEGF-A (Pearson correlation: $\mathrm{r}=0.737, \mathrm{p}<0.01, \mathrm{n}=90$ ) (Figure 1B). Thus, up-regulation of L-FABP is apparently correlated with HCC VEGF-A expression.

\section{L-FABP induces VEGF-A expression and increases angiogenic potential in immortalized Hus and Huh7 cells}

L-FABP expression was analyzed in various cell lines, including Hus (normal hepatocytes) and HCC (HepG2, Hep3B, Huh7, and PLC/PRF/5) cells. L-FABP was highly expressed in HepG2 and Huh7 cells, which also showed strong VEGF-A expression levels in their cytosol (Figure 2A) and culture medium (Supplementary Figure 1), in addition to higher angiogenic potential (Figure 2B).

To examine the effects of L-FABP on VEGF-A expression, we generated L-FABP-overexpressing stable clones with Hus cells and we used Huh7 cells to produce L-FABP shRNA knockdown clones. As shown in Figure 2C, VEGF-A expression (at both the mRNA and protein levels) was higher in Hus/L-FABP cells than in control cells, whereas the expression of VEGF-A decreased markedly in Huh7/L-FABP shRNA cells relative to the control (Supplementary Figure 2A).

Angiogenesis was also significantly higher in Hus/ L-FABP cells than in control cells (Figure 2D), i.e., it decreased in Huh7/L-FABP shRNA cells (Supplementary Figure 2B). To further examine whether L-FABP promotes angiogenesis in vivo, we performed a Matrigel plug assay in NOD/SCID mice by using Hus/L-FABP (Figure 2E, a) or Huh7/L-FABP shRNA cells (Supplementary Figure 2C). Anti-CD31 immunohistochemical staining indicated that L-FABP-overexpressed cells promoted angiogenesis by inducing neovascular formation in Matrigel (Figure 2E, b, p < 0.05).

\section{L-FABP interacts with VEGFR2 in membrane rafts}

Previous studies reported that some FABPs, such as H-FABP or B-FABP, could interact with membraneassociated receptors, including integrin or dopamine D2 receptor [9-11], and that L-FABP may associate with cell membranes or membrane proteins $[5,12]$. A recent study also revealed that L-FABP was significantly associated with hepatocyte plasma membrane cholesterol-rich microdomains [13]. Thus, we examined whether L-FABP interacted with membrane receptors as follows. We proposed that L-FABP was associated with membrane receptors and, by using an alignment of amino acid sequences thought to interact with FABP, we identified the consensus sequence most likely to interact with L-FABP: WKIGFXKRLXXVXXXI (Supplementary Figure 3). By comparing the consensus sequence to that of membrane receptors, we found that the kinase domain of VEGFR2 potentially interacted with L-FABP. A co-immunoprecipitation assay with primary antibodies against VEGFR2 or L-FABP, in addition to blotting with L-FABP or VEGFR2, showed that L-FABP could interact with VEGFR2 (Figure 3A). To further confirm this result, we purified L-FABP recombinant protein using a nickel column (Supplementary Figure 4), and performed an overlay assay (far-western blot analysis) to study the interaction between L-FABP and VEGFR2. We found that L-FABP directly interacted with the VEGFR2 intracellular domain (aa 789 to end) in a cell-free system. Moreover, the binding curve revealed that the $\mathrm{Kd}$ (dissociation constant) for the interaction of VEGFR2 and L-FABP was $0.25 \mathrm{nM}$ (Figure 3B).

Confocal microscopy images of Hus/L-FABP cells revealed that L-FABP was located in both the membrane and cytosol, whereas VEGFR2 was expressed mainly at the membrane. Co-localization of L-FABP and VEGFR2 in the apical membrane was observed in Hus/L-FABP cells (Figure 3C), but disappeared in Huh7 cells with L-FABP knockdown (Supplementary Figure 5). Sucrose gradient ultracentrifugation was conducted to confirm this co-localization. Fractions with lipid rafts of Hus/L-FABP cells were identified by flotillin-2, a lipid raft marker; moreover, not only L-FABP and VEGFR2 but also membrane associating signal transduction proteins (e.g., PI3K (p85), p-Akt/Akt, p-Src/Src, p-FAK/FAK) showed increased distribution in membrane rafts (Figure 3D). Taken together, these results suggest that overexpressed L-FABP was not only associated with membrane VEGFR2 but also activated its downstream signal transduction.

\section{L-FABP increases VEGFR2/Src phosphorylation and cell migration via the FAK/cdc42 pathway}

The VEGFR2/Src pathway reportedly affects cancer cell migration by activating FAK and Rho-GTPase 

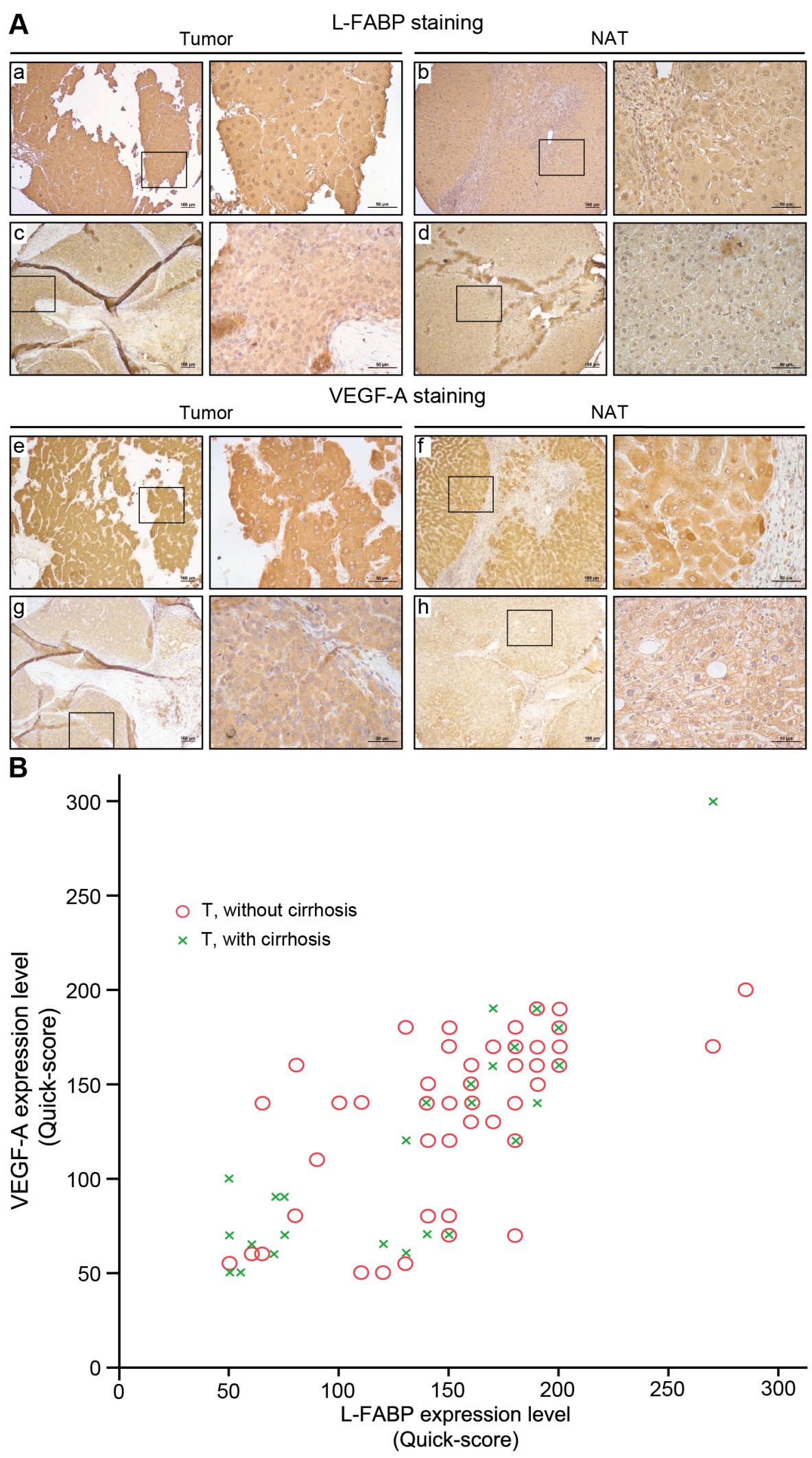

Figure 1: Expression of L-FABP and VEGF-A in tissues obtained from HCC patients. Protein expression was assessed in 90 HCC cases using immunohistochemical staining of paired normal (NAT) and tumor tissues. A. Staining of L-FABP and VEGF-A was observed in tumor tissues (L-FABP: a and c; VEGF-A: e and g) and their paired normal adjacent tissues (L-FABP: $b$ and d; VEGF-A: $\mathrm{f}$ and h). Staining intensity: a and e, strong; b, c, f, and g, moderate; d and h, weak. B. Positive correlation between L-FABP and VEGF-A expression in 90 HCC tissues with and without cirrhosis (Pearson's correlation coefficient, $r=0.737$; $\mathrm{p}<0.01$ ). 
Table 1: Correlation between L-FABP and VEGF-A protein expression in tissue pairs from $90 \mathrm{HCC}$ patients

\begin{tabular}{|c|c|c|c|c|c|c|c|c|c|}
\hline Intensity $^{\mathrm{a}}$ & & $\begin{array}{c}\text { NAT, } \mathbf{N} \\
(\%)\end{array}$ & $\begin{array}{c}\text { HCC without } \\
\text { cirrhosis, } \mathrm{N} \\
(\%)\end{array}$ & $\begin{array}{c}\text { HCC with } \\
\text { cirrhosis, } \\
\text { N }(\%)\end{array}$ & P value $^{b}$ & P value ${ }^{c}$ & $P_{\text {value }}{ }^{d}$ & $P_{\text {value }}{ }^{e}$ & Pvalue $^{\mathrm{f}}$ \\
\hline \multirow[t]{3}{*}{ L-FABP } & 1 & $15(44.1)$ & $8(23.5)$ & $11(32.4)$ & 0.012 & 0.028 & 0.027 & 0.040 & 0.086 \\
\hline & 2 & $72(55.0)$ & $40(30.5)$ & $19(14.5)$ & & & & & \\
\hline & 3 & $3(20.0)$ & $9(60.0)$ & $3(20.0)$ & & & & & \\
\hline \multirow[t]{3}{*}{ VEGF-A } & 1 & $25(48.1)$ & $12(23.1)$ & $15(28.8)$ & 0.025 & 0.563 & 0.360 & 0.037 & 0.017 \\
\hline & 2 & $65(51.2)$ & $45(35.4)$ & $17(13.4)$ & & & & & \\
\hline & 4 & $0(0.0)$ & $0(0.0)$ & $1(100.0)$ & & & & & \\
\hline
\end{tabular}

Abbreviations: HCC, hepatocellular carcinoma; OR, odds ratios; CI, confidence interval; N, number.

a Intensity: 0, negative; 1, weak positive; 2, moderate positive; 3, strong positive; 4, very strong positive.

${ }^{\mathrm{b}}$ Chi-square test, NAT vs HCC without cirrhosis vs HCC with cirrhosis.

${ }^{c}$ Chi-square test, NAT vs HCC with or without cirrhosis.

${ }^{\mathrm{d}}$ Chi-square test, NAT vs HCC without cirrhosis.

${ }^{\mathrm{e}}$ Chi-square test, NAT vs HCC with cirrhosis.

${ }^{\mathrm{f}}$ Chi-square test, $\mathrm{HCC}$ without cirrhosis vs $\mathrm{HCC}$ with cirrhosis.

[14-16]. In Hus/L-FABP cells, the phosphorylation of VEGFR2, Src, and FAK increased significantly (Figure 4A and $4 \mathrm{~B}$ ) and the activity of cdc42 was significantly increased (Figure $4 \mathrm{C}, \mathrm{p}<0.001$ ). A wound-healing assay (Figure 4D) and Boyden chamber-based migration assay (Figure 4E) of 2D and 3D migration activity, respectively, showed that migration activity was higher in Hus/LFABP cells than in control cells. To determine whether or not L-FABP-induced cell migration occurred via cdc42, plasmids expressing different variants of cdc42, including wild-type (WT), constitutively active (CA), and dominant negative (DN) cdc42, were transfected into the cells. Phalloidin staining (Supplementary Figure 6A) and a transwell assay (Supplementary Figure 6B) showed that the activity of cdc 42 strongly affects actin rearrangement and cell migration induced by L-FABP, which was in line with our previous findings. In contrast, L-FABP-knockdown clones showed significantly reduced 3D migration activity (Supplementary Figure 2D, p $<0.001$ ). Sorafenib (VEGFR2 inhibitor) or PP1 (Src inhibitor) treatments significantly inhibited $3 \mathrm{D}$ migration activity in Hus/L-FABP cells (Figure 4F, p $<0.001$ ). Moreover, knockdown of L-FABP in Hus/L-FABP-stable clones reversely decreased their 3D migration activity (Supplementary Figure 7C). These results suggest that VEGFR2/Src signaling participates in L-FABP-induced migration activity via the $\mathrm{FAK} / \mathrm{cdc} 42$ pathway.

\section{L-FABP induces VEGF-A expression via the Akt/

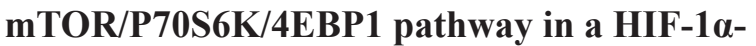 dependent manner}

Based on the results shown in Figure 3D, and previous studies suggesting that Akt activation increases
VEGF-A expression and is necessary and sufficient to regulate HIF-1 $\alpha$ and VEGF expression in various human cancer cells [17-19], we postulated that the signal transduction of L-FABP-mediated VEGF-A expression was activated through the Akt pathway. In western blot analysis, we observed activation of the Akt/mTOR/P70S6K/4EBP1 pathway in Hus/L-FABP cells (Figure 5A). The expression of VEGF-A mRNA can apparently be regulated in an HIF-1 $\alpha$-dependent manner [20, 21]. Here, VEGF-A mRNA expression was significantly increased in L-FABP-overexpressing cells as mentioned in Figure 2C, and HIF-1 $\alpha$ levels significantly increased in the nucleus fraction of Hus/L-FABP cells (Figure 5B, p $<0.05$ ). Inhibition of the PI3K/Akt pathway by treatment with LY294002 (PI3K inhibitor) decreased the expression of HIF-1 $\alpha$ and VEGF-A (Supplementary Figure 8). These data suggest that L-FABP induced VEGF-A expression through Akt activation, and that this process could be regulated by HIF- $1 \alpha$.

To confirm our findings, full-length and successive $5^{\prime}$ deletion (D1-D3) constructs of the VEGF-A gene promoter were cloned into pGL4.22 luciferase reporter vectors, and a luciferase reporter assay was conducted (Figure 5C, a). Results showed that VEGF-A transcriptional activity was elevated 16.5 -fold in L-FABP-overexpressing Hus cells compared with that in control cells, whereas deletion of the HIF-1 $\alpha$ binding site (D1-D3) reduced this activity to $\sim 2.5$ fold that of the control (Figure 5C, b). Additionally, the chromatin immunoprecipitation assay demonstrated that the association between HIF-1 $\alpha$ and the VEGF-A promoter was enhanced in Hus/L-FABP cells (Figure 5D). To further address the regulation of VEGF-A expression, Hus/L-FABP cells were treated with rapamycin (mTOR inhibitor) or cyclohexamide (translation inhibitor); 


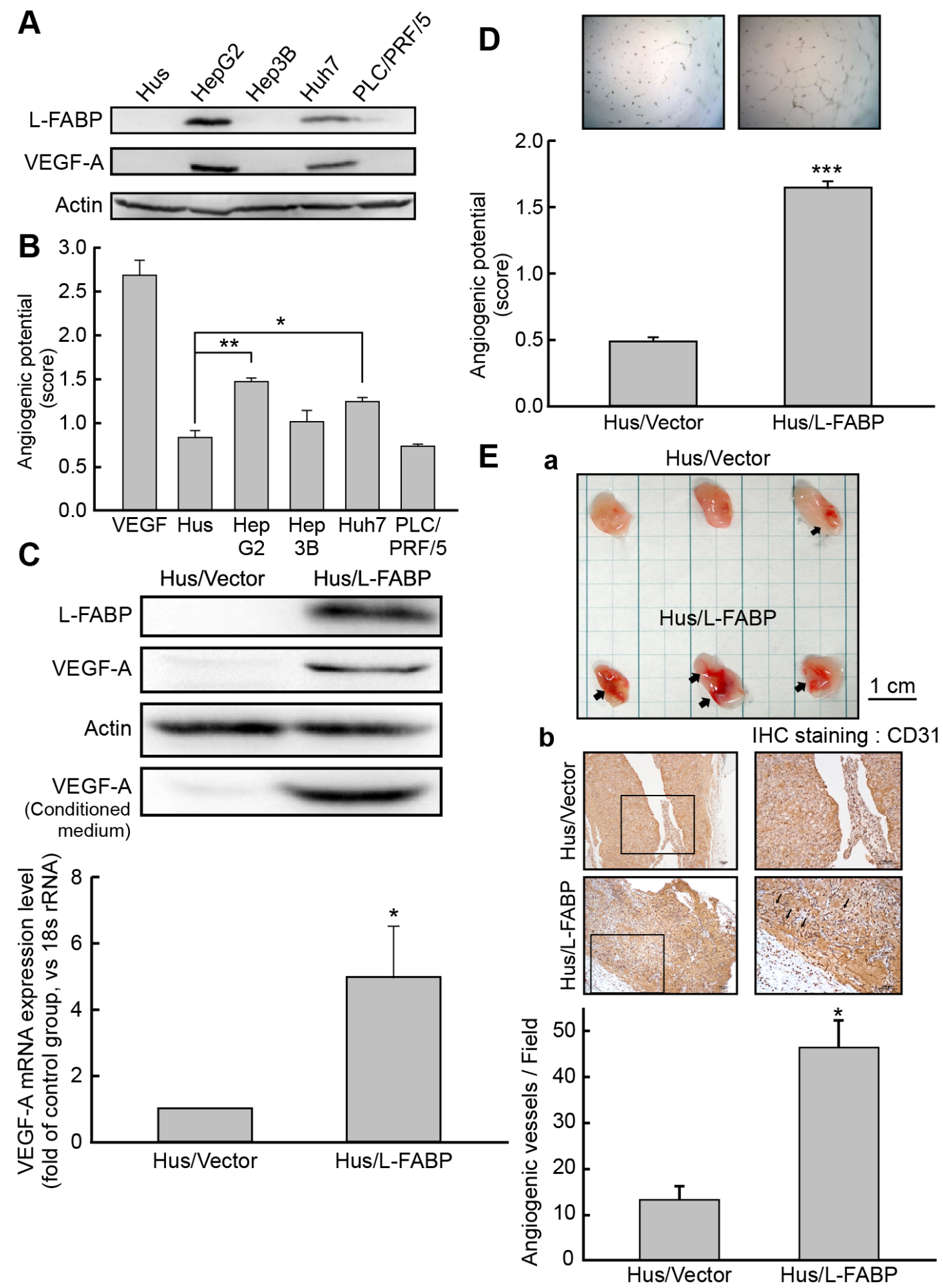

Figure 2: L-FABP promotes VEGF-A expression and angiogenic activity of liver cells. A. Western blot analysis for L-FABP expression in normal immortalized hepatocyte (Hus) and hepatocellular carcinoma (HepG2, Hep3B, Huh7 and PLC/PRF/5) cell lines. B. Angiogenic potential (score: see In vitro tube formation assay in Methods for details) of Hus, HepG2, Hep3B, Huh7, and PLC/PRF/5 cells. ${ }^{* *} \mathrm{p}<0.001$ versus Hus cells. C. Western blotting analysis of L-FABP and VEGF-A expression in Hus/L-FABP and Hus/Vector (vector-only control) cells. ${ }^{*} \mathrm{p}<0.05$ versus Hus/Vector control. D. In vitro angiogenic potential (score: see panel B) of Hus/L-FABP and Hus/Vector cells. Angiogenic vascular tube was imaged at 8 h. ${ }^{* * *} \mathrm{p}<0.001$ versus Hus/Vector control. E. In vivo angiogenic activity of Hus/L-FABP and Hus/Vector cells assessed using a Matrigel plug assay. a: Matrigel plugs recovered from mice injected with Hus/Vector and Hus/L-FABP cells. Arrows indicate infiltration of blood vessels. b: Immunohistochemical (IHC) staining of CD31 (angiogenesis marker) in Matrigel plugs showed that Hus/L-FABP promoted angiogenesis, and the positively stained vessels are indicated by arrows. $* \mathrm{p}<0.05$ versus Hus/Vector control $(\mathrm{n}=3)$. 
consequently, decreased VEGF-A expression (Figure 5E, a) and concentration-dependent inhibition of angiogenic potential (Figure 5F) were observed. Treatment of Hus/ Vector cells with the proteasome inhibitor MG132 also indicated that L-FABP-induced VEGF-A expression did not occur via inhibition of protein degradation (Figure 5E, b). Taken together, these data suggest that L-FABP-induced VEGF-A expression was regulated via the Akt/mTOR/ P70S6K/4EBP1 pathway in a HIF-1 $\alpha$-dependent manner.

\section{L-FABP promotes tumor growth and metastasis in vivo}

The role of L-FABP in tumorigenesis was examined in immune-deficient NOD/SCID mice. At Day 50, tumor growth was significantly enhanced in mice injected with Hus/L-FABP cells, whereas no significant tumor growth was observed in Hus/Vector cell-injected (control) mice (Figure 6A). The level of VEGF-A in the serum of
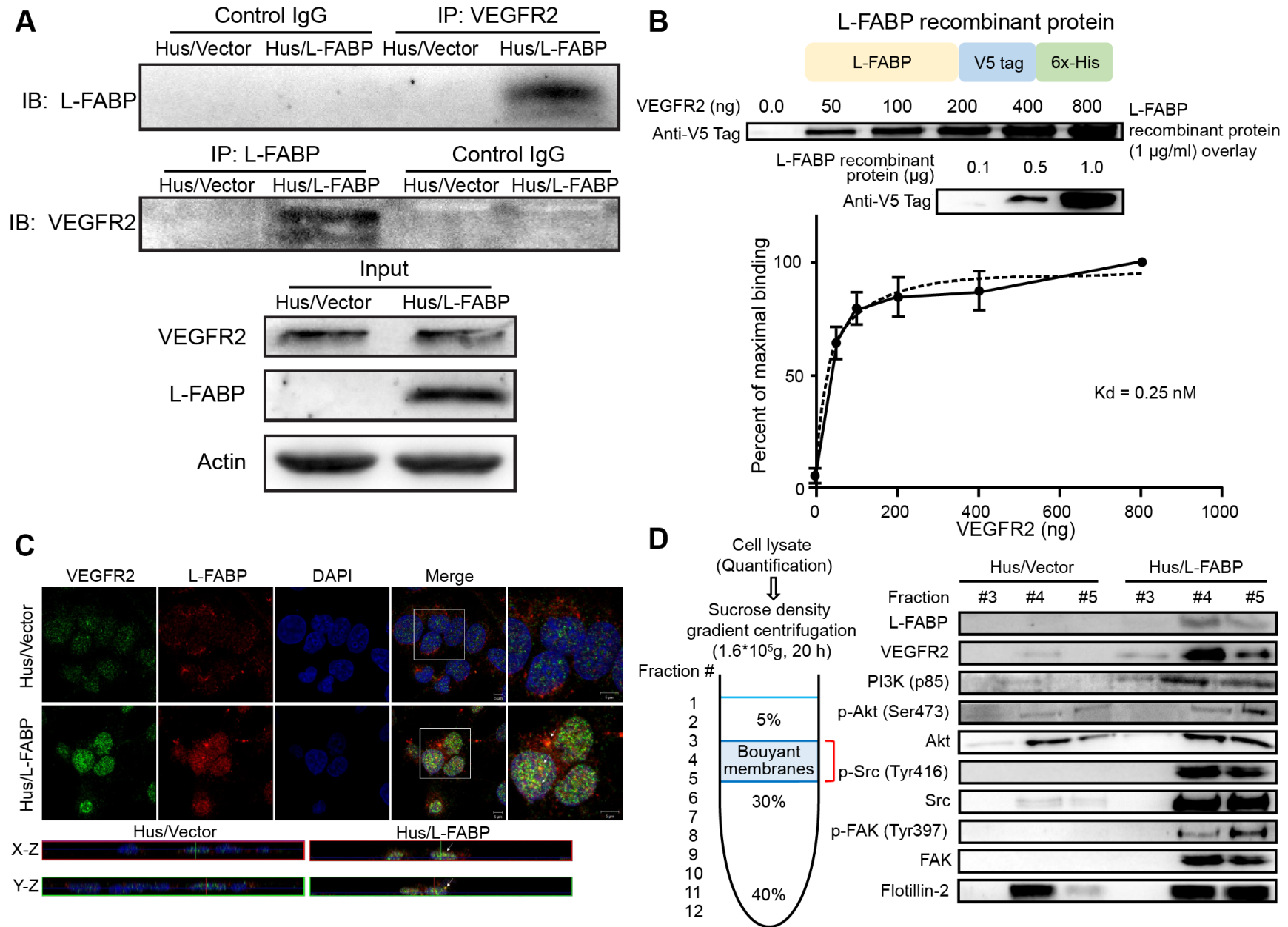

Figure 3: L-FABP associates with VEGFR2 on membrane rafts. A. Left: Hus/L-FABP and Hus/Vector (vector-only control) cells were subjected to either immunoprecipitation (IP) with a VEGFR2 antibody followed by blotting with L-FABP or IP with an L-FABP antibody followed by blotting with VEGFR2. Right: Cell lysates $(50 \mu \mathrm{g})$ were immunoblotted as an input control. B. L-FABP/V5-tagged recombinant protein was purified by Ni-NTA resin and subjected to SDS-PAGE to determine the purity as shown in the top photo. The overlay assay (far western blot analysis) was performed to estimate the affinity of the interaction between the VEGFR2 intracellular domain and L-FABP. PVDF membranes containing 0.5 to $8.0 \mu \mathrm{g}$ of VEGFR2 recombinant protein (aa 789 to end) were incubated with L-FABP/ V5-tagged recombinant protein $(1 \mu \mathrm{g} / \mathrm{ml})$ for 12 hours. The specific binding between L-FABP and VEGFR2 increased obviously between 0 to $2 \mu \mathrm{g}$, and maximal binding was observed at $8 \mu \mathrm{g}$. The binding observed at the other concentrations was expressed as a percentage of the maximal binding within each experiment, and the Kd for binding between L-FABP and VEGFR2 was calculated as $0.25 \mathrm{nM}$. C. Threecolor confocal images of cells that were fixed and stained with antibodies against L-FABP and VEGFR2. Signals: green, VEGFR2-Alexa 488; red, L-FABP-Alexa 568; and blue, DAPI. Magnification: 63×. The bottom photos show the X-Z and Y-Z optical sections, respectively, of Hus/Vector and Hus/L-FABP cells. Arrows indicate the co-localization of VEGFR2 and L-FABP in the apical membrane. D. Membrane localization of L-FABP, VEGFR2, PI3K (p85), phospho-Akt (Ser473), Akt, phosho-Src (Tyr416), Src, FAK, and phosho-FAK (Tyr397) in Hus/L-FABP or Hus/Vector cells. Membrane rafts were determined by using sucrose gradient-based ultracentrifugation and analyzed with western blotting (fraction \#3-5). 
mice also increased 2.8-fold in the Hus/L-FABP group relative to the control group (Figure 6B). Additionally, immunohistochemical staining of CD31 indicated that L-FABP induced angiogenesis in vivo (Figure 6C). In an in vivo tumor metastasis assay, the number of metastatic nodules formed in the lungs of NOD/SCID mice after 60 days was 3.9-fold higher in the Hus/LFABP-injected group relative to the control group (Figure $6 \mathrm{D})$, and angiogenic vessel formation was increased in these nodules (Figure 6E). The results of these in vivo experiments support the correlation between L-FABP and VEGF-A expression.

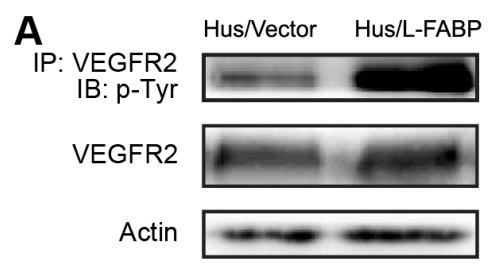

B
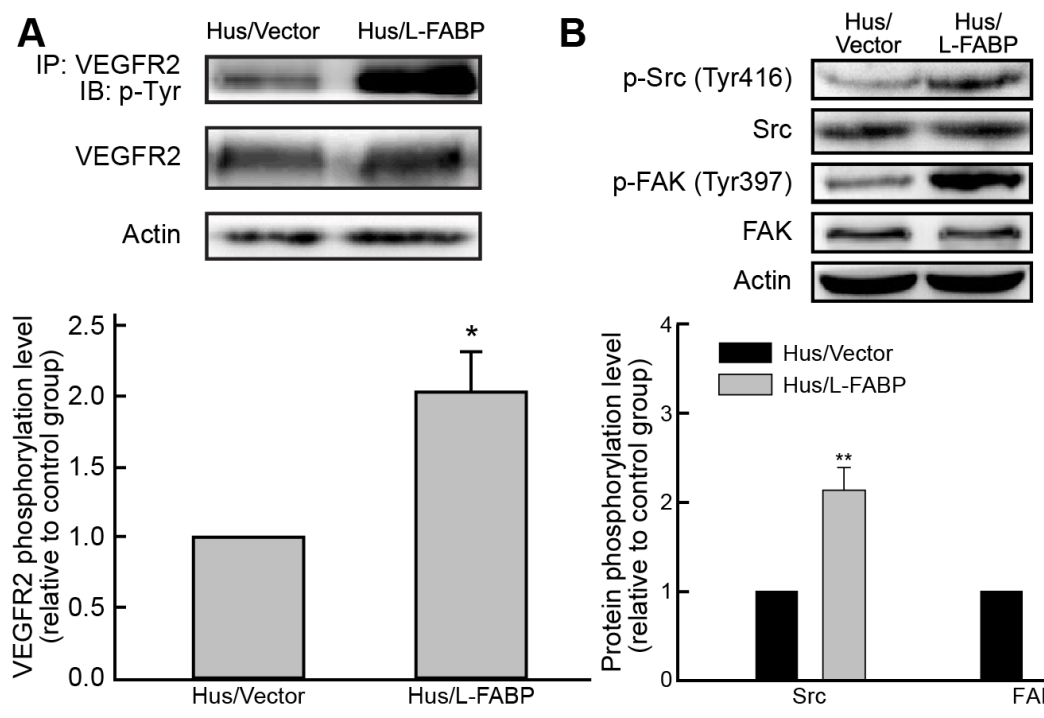

D
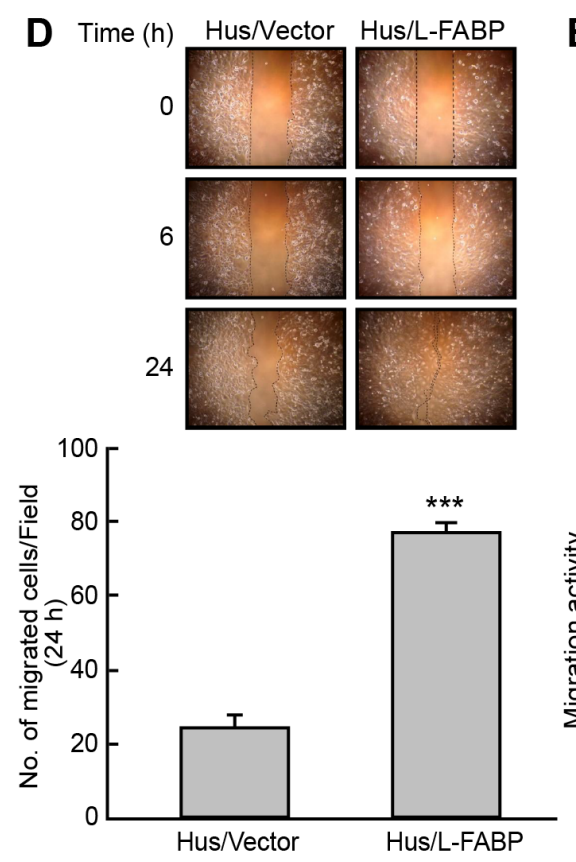

$E$

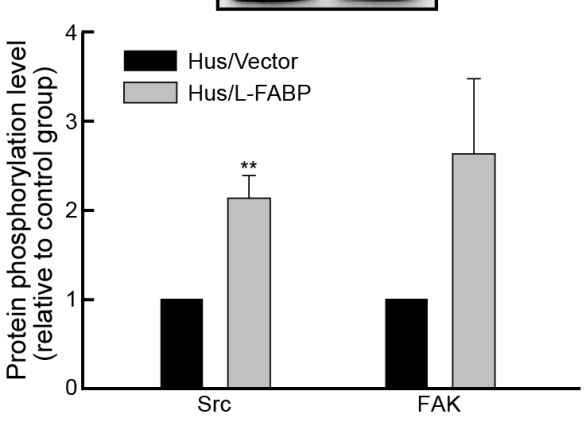

\section{E}
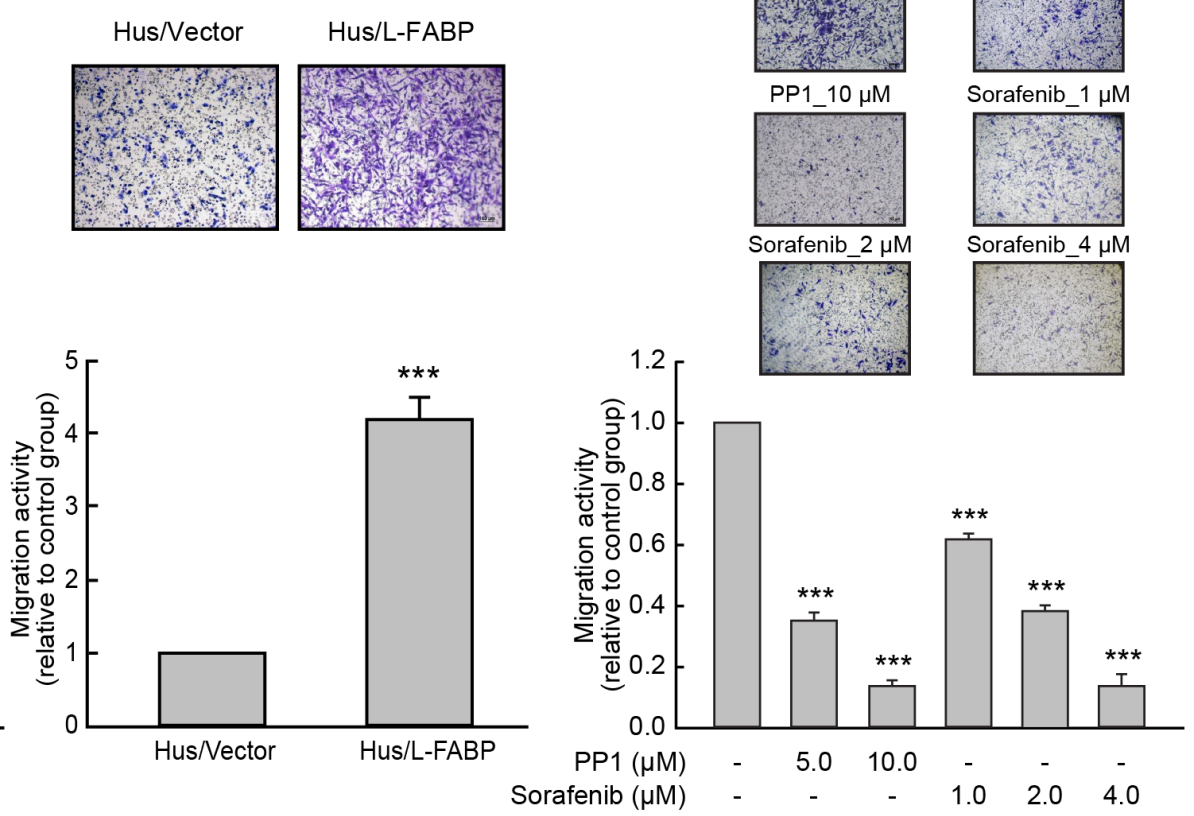

Figure 4: L-FABP increases cell migration activity via VEGFR2/Src signaling and the FAK/cdc42 pathway. A. Phosphorylation of VEGFR2 in Hus/L-FABP and Hus/Vector (vector-only control) cells assessed by immunoprecipitation (IP) with a VEGFR2 antibody and blotting with a phospho-tyrosine antibody. ${ }^{*} \mathrm{p}<0.05$ versus Hus/Vector control. B. Phosphorylation of Src (Tyr416) and FAK (Tyr397) in Hus/L-FABP and Hus/Vector cells analyzed by western blotting. **p $<0.01$ versus Hus/Vector control. C. Small GTPase binding in Hus/L-FABP or Hus/Vector cells. Active cdc42 and Rac1, but not RhoA, were detected by western blotting analysis. $* * * \mathrm{p}<0.001$ for cdc42 activity versus Hus/Vector control. D. Wound-healing migration (2D migration activity) of Hus/L-FABP and Hus/Vector cells over 24 h. ${ }^{* *} \mathrm{p}<0.001$ versus Hus/Vector control. E. Migration activity of Hus/L-FABP and Hus/Vector cells seeded onto Boyden chambers and allowed to migrate toward $10 \%$ serum-containing medium for $16 \mathrm{~h}$. ***p $<0.001$ versus Hus/Vector control. F. Migration activity of Hus/L-FABP cells treated with PP1 (Src inhibitor: 5 or $10 \mu \mathrm{M}$ ) or Sorafenib (VEGFR2 inhibitor: 1, 2, or $4 \mu \mathrm{M})$ for 16 h. $* * * \mathrm{p}<0.001$ versus DMSO-treated control group. 


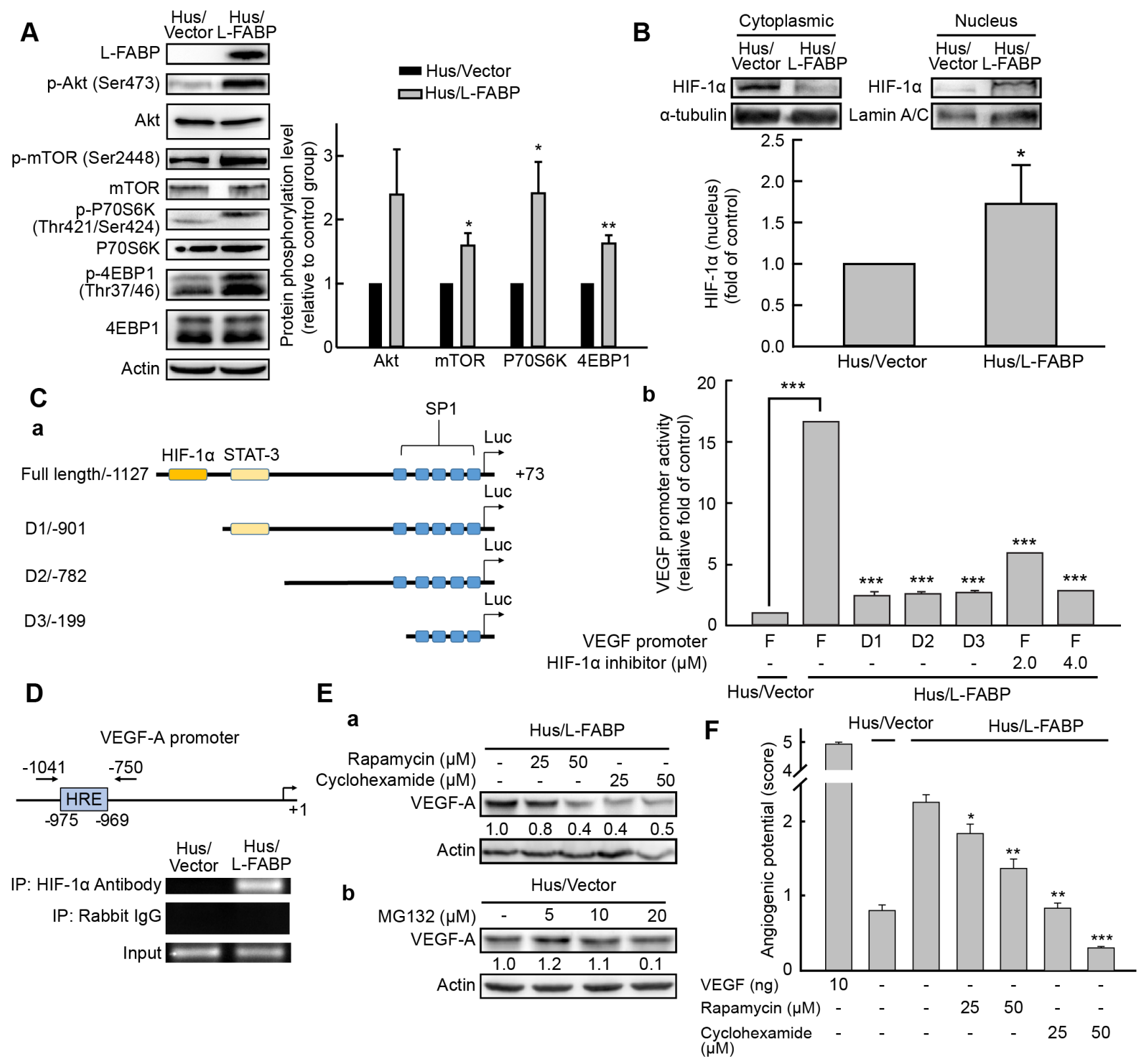

Figure 5: L-FABP-promoted VEGF-A expression is regulated by HIF-1 $\alpha$ via the Akt/mTOR/P70S6K/4EBP1 pathway. A. Phosphorylation of Akt (Ser473), mTOR (Ser2448), P70S6K (Thr421/Ser424), and 4EBP1 (Thr37/46) in Hus/L-FABP and Hus/Vector (vector-only control) cells analyzed by western blotting. ${ }^{*} \mathrm{p}<0.05$ and $* * \mathrm{p}<0.01$ versus Hus/Vector control. B. Nucleus and cytoplasmic localization of HIF- $1 \alpha$ in Hus/L-FABP cells. Loading controls were $\alpha$-tubulin and lamin A/C for cytoplasm and nucleus, respectively. HIF$1 \alpha$ levels increased $\sim 1.7$-fold in Hus/L-FABP relative to the control group: ${ }^{*} p<0.05$. C. a: Diagram of the receptor constructs for the fulllength VEGF-A promoter and deletion mutants (D1-D3). b: The luciferase activity of cell extracts was analyzed using a luciferase reporter assay (bar graph). ${ }^{* * *} \mathrm{p}<0.001$ versus Hus/Vector control. D. Chromatin immunoprecipitation assay was performed to determine the amount of HIF-1 $\alpha$ binding to the VEGF-A promoter; rabbit IgG served as a negative control, and the input served as a positive control. E. a: Western blot analysis of Hus/L-FABP cells treated with rapamycin (mTOR inhibitor: 25 or $50 \mu \mathrm{M}$ ) or cyclohexamide (translation inhibitor: 25 or $50 \mu \mathrm{M}$ ) for $12 \mathrm{~h}$. b: Hus/Vector cells treated with MG132 (proteasome inhibitor: 5, 10, or $20 \mu \mathrm{M}$ ) for $24 \mathrm{~h}$. F. In vitro angiogenic activity (score: see In vitro tube formation assay in Methods for details) of Hus/L-FABP and Hus/Vector cells treated with rapamycin or cyclohexamide (doses identical to those in D) for $12 \mathrm{~h} . * * * \mathrm{p}<0.001$ versus Hus/Vector control. 


\section{Cholesterol association and membrane interaction properties are essential for L-FABP- induced cell migration and angiogenesis}

Previous studies suggested that L-FABP mutations prevent fatty acid or cholesterol uptake and even alter membrane structure [22-26]. Thus, to investigate the effects of L-FABP on the membrane of L-FABP- overexpressing cells, we used site-directed mutagenesis to generate L-FABP-overexpressing stable clones with substitutions of various functional amino acids in Hus cells. Three mutants, namely F3W, K31E, and T94A, exhibited reduced VEGF-A expression relative to the wildtype (Figure 7A), and these mutants showed significantly decreased angiogenic potential (Figure 7B). However, migration activity was significantly reduced in $\mathrm{K} 31 \mathrm{E}$ and

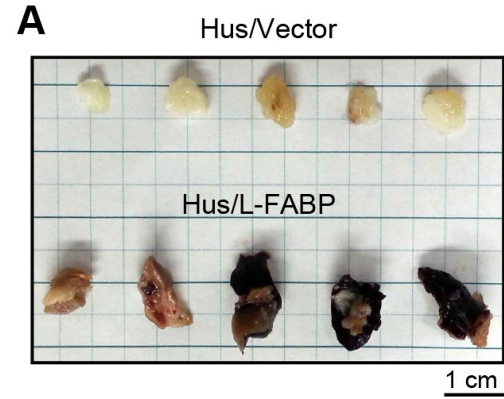

C
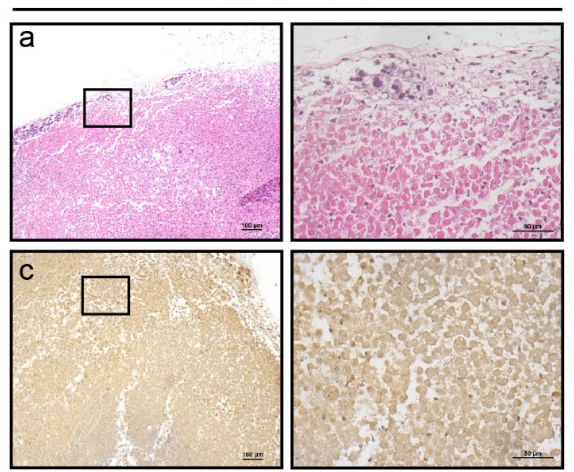

E Hus/Vector
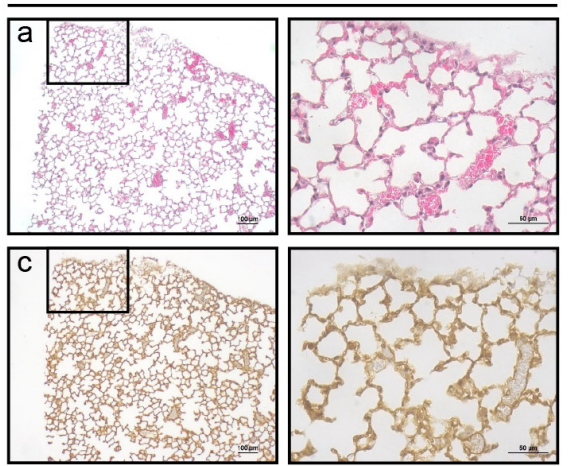
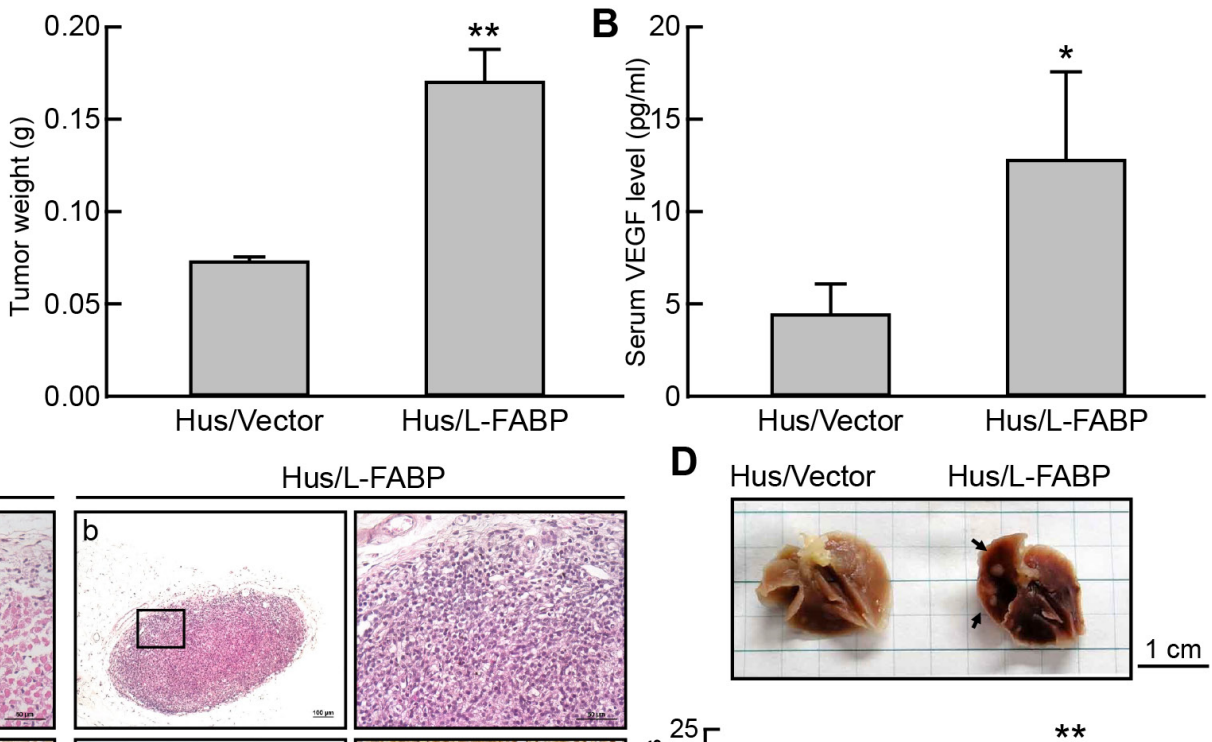

D Hus/Vector

Hus/L-FABP
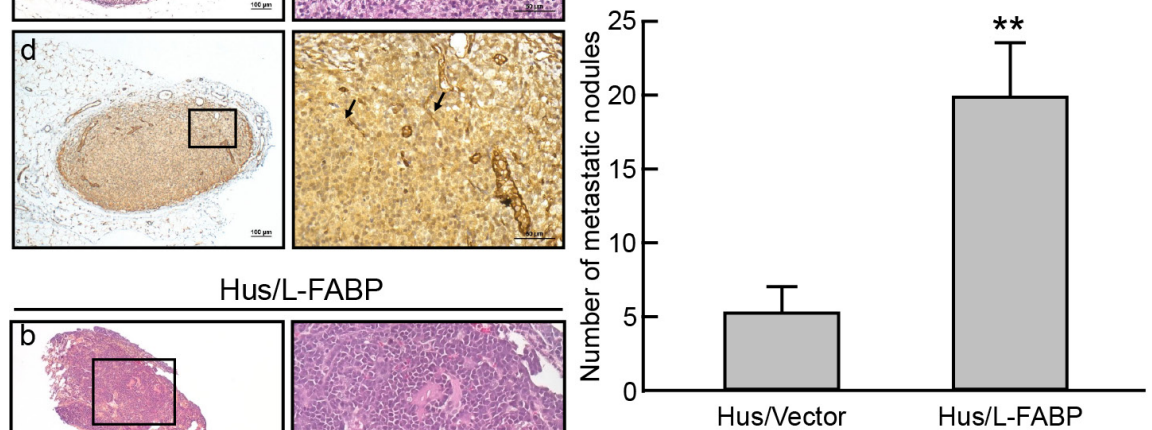

Figure 6: L-FABP promotes tumor growth and metastasis in vivo. Hus/L-FABP or Hus/Vector (vector-only control) cells $\left(2 \times 10^{6}\right)$ was subcutaneously injected into the hind limbs of NOD/SCID mice, and the resulting in situ tumors were removed after 8 weeks for analysis. A. Representative photograph and average weight of tumors ( $\mathrm{n}=5$ per group). ** $\mathrm{p}<0.01$ versus Hus/Vector control. B. VEGF-A content in the serum of treated mice. ${ }^{*} \mathrm{p}<0.001$ versus Hus/Vector control. C. Stained tumor sections from Hus/L-FABPand Hus/Vector-injected mice. H\&E staining ( $a$ and b) and anti-CD31 antibody immunohistochemical staining (c and d). The positive staining indicated by the arrows shows strong angiogenic activity in the Hus/L-FABP-injected group. D. Metastatic activity of Hus/LFABP and Hus/Vector cells $\left(5 \times 10^{6}\right)$ in a lung metastasis model (NOD/SCID mice). After 10 weeks, the lungs were excised from mice (see photograph), and metastatic nodules (indicated by arrows) were counted ( $n=5$ per group). ${ }^{* *} \mathrm{p}<0.01$ versus Hus/Vector control. E. Angiogenesis activity in metastatic nodules was assessed via H\&E staining (a and b) or anti-CD31 immunohistochemical staining (c and d), and positive staining is indicated by arrows. 
A

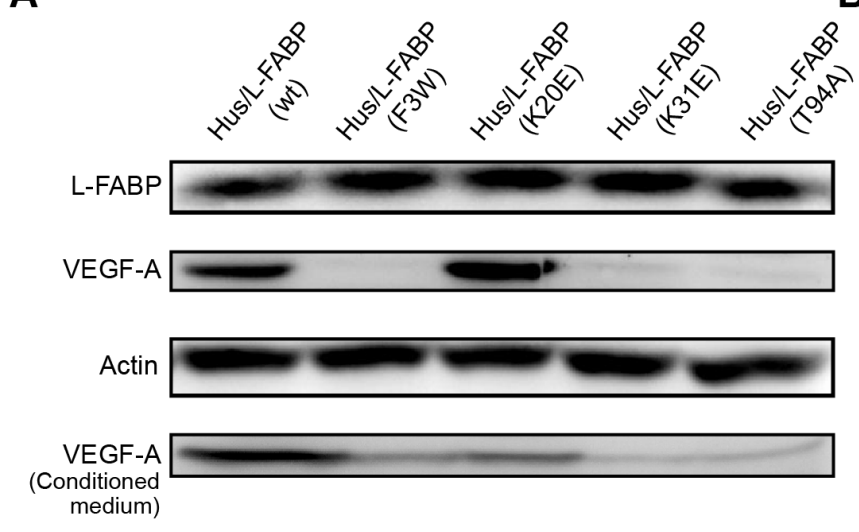

C
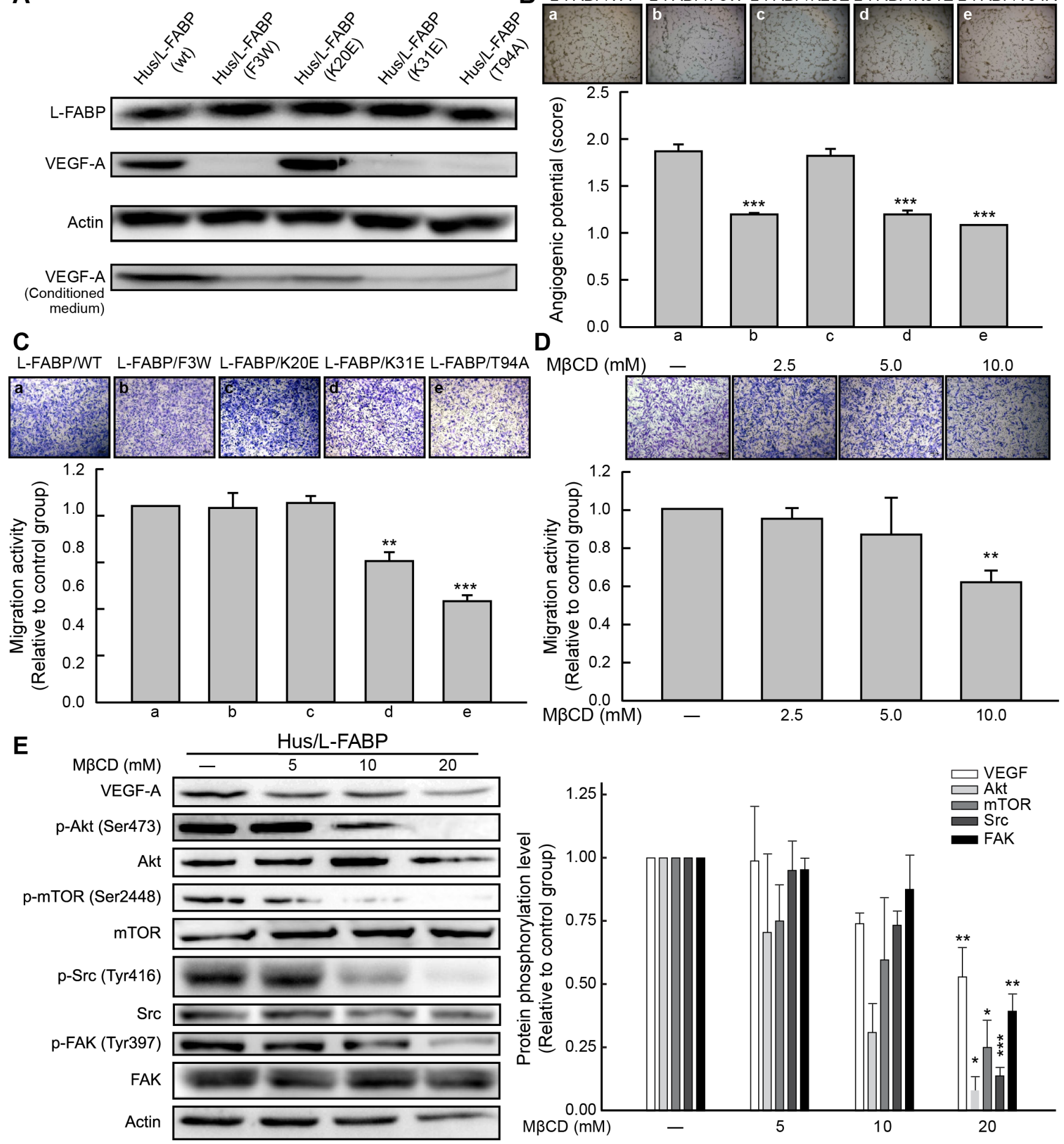

Figure 7: Cholesterol binding properties are essential for L-FABP-induced cell migration and angiogenesis. A. Western blotting analysis of L-FABP and VEGF-A expression (both intracellular and extracellular levels) in various mutants of L-FABPoverexpressed stable cells generated by site-directed mutagenesis B. In vitro angiogenic activity (score: see In vitro tube formation assay in Methods for details) of mutants. Images represent amino acid substitutions: (a) L-FABP (wild type), (b) L-FABP (F3 to W), (c) L-FABP (K20 to E), (d) L-FABP (K31 to E), and (e) L-FABP (T94 to A). ***p $<0.001$ versus wild-type. C. Migration activity of the mutants. Images (a-e) represent the amino acid substitutions described in (B). ${ }^{*} \mathrm{p} p<0.01,{ }^{* * *} \mathrm{p}<0.001$ versus wild-type. D. Migration activity of Hus/L-FABP cells treated with M $\beta C D$ (cholesterol depletion agent: 5, 10, or $20 \mathrm{mM}$ ) for $12 \mathrm{~h}$. **p $<0.01$ versus water-treated control group. E. Western blot analysis of Hus/L-FABP cells treated with $\operatorname{MBCD}(5,10$, or $20 \mathrm{mM})$ for 6 h. ${ }^{*} \mathrm{p}<0.05,{ }^{* *} \mathrm{p}<0.01,{ }^{* * *} \mathrm{p}<0.001$ versus water-treated control group. 
T94A mutants only (Figure 7C, p < 0.01). T94A is the most common mutation in Europeans, and it is known to affect fatty acid and cholesterol uptake as a loss-offunction mutation [24, 26]. For further substantiation, we reduced the membrane cholesterol content in Hus/L-FABP cells by using $\mathrm{M} \beta \mathrm{CD}$ (a cholesterol depletion reagent). VEGF expression, migration activity, and related signaling were all down-regulated in M $\beta C D$-treated Hus/L-FABP cells (Figure 7D and 7E). Overall, our data suggest that the oncogenic activity of L-FABP was associated with its membrane-binding properties.

\section{DISCUSSION}

$\mathrm{HCC}$ is characterized by its aggressiveness and angiogenic capability; thus, the angiogenic factor VEGF is considered to be a target for HCC therapy $[1,3]$. Here, we reported for the first time that overexpression of L-FABP plays an important role in VEGF-A expression and cell migration in HCC. Furthermore, we demonstrated that L-FABP associates with VEGFR2 in the cell membrane, which leads to activation of VEGFR2-related signaling (i.e., Src/FAK/cdc42 and Akt/mTOR/HIF-1 $\alpha$ signaling). Additionally, we showed that the T94A mutation of L-FABP, which is related to cholesterol association activity, significantly decreases the angiogenic potential and migration activity of L-FABP-overexpressing cells.

It has been suggested that L-FABP promotes the growth of hepatocytes and protects cells from ROS via its anti-oxidative activity, which is related to methionine and cysteine [27, 28]. Other studies have also found evidence for a correlation between L-FABP and VEGF $[7,8]$. A recent study reported that FABP4 (A-FABP) plays an important role in regulating the function of VEGF function and promoting proliferation of HUVEC cells [29]; however, the link between L-FABP and tumor malignancy is still unclear. Here, we found a significant increase in L-FABP expression in tumor tissue relative to normal adjacent tissue in $90 \mathrm{HCC}$ patients, and L-FABP and VEGF-A expression was positively correlated in these tissues. L-FABP and VEGF-A expression was also higher in malignant HCC cell lines (HepG2 and Huh7) than in immortalized normal hepatocytes (Hus cells). Given these findings, we propose that L-FABP may participate in VEGF-A expression in HCC. Further investigation supported this hypothesis, with stable clones of Hus/LFABP cells exhibiting increased VEGF-A expression and angiogenic potential both in vitro and in vivo. Additional evidence was provided by our experiments involving L-FABP knockdown in Huh7 cells, L-FABPoverexpressing Hus cells (Supplementary Figure 7A and 7B), and HepG2 cells (Supplementary Figure 9A). A previous study suggested that VEGF is essential for HCC cell migration [30]. We found that the migration activity of Hus/L-FABP cells increased significantly compared with that of control cells. Knockdown of L-FABP in
Huh7, L-FABP-overexpressing Hus cells, and HepG2 cells (Supplementary Figure 9B) significantly decreased migration activity relative to control groups. Taken together, these results suggest that L-FABP overexpression plays a critical role in the angiogenic potential and migration activity of $\mathrm{HCC}$ cells, and that this effect can be reversely regulated using RNA knockdown technology.

Previous studies have clarified the binding mechanism of VEGF-A to VEGFR2, which consists of one VEGF-A dimer binding to one VEGFR2 homo- or hetero-dimer. The Kd of VEGF-A and VEGFR2 was also calculated by different methods (for ITC, $\mathrm{Kd}=18 \pm 5.2$ $\mathrm{nM}$, for SPR, $\mathrm{Kd}=36.7 \pm 5.9 \mathrm{nM}$ ) [31]. In the present study, we found that L-FABP interacts with VEGFR2 in cells overexpressing L-FABP. We also determined that the $\mathrm{Kd}$ of L-FABP and VEGFR2 (intracellular domain) in a cell-free system was $0.25 \mathrm{nM}$, suggesting that L-FABP could be a cytosolic interacting protein for VEGFR2. Because the detailed binding mechanism remains unclear, further studies should be performed using the SPR system (Biacore) or protein crystallization.

In an early study, L-FABP was reported to interact with the cell membrane [6]. However, most subsequent studies have focused on its biological function in transporting fatty acids and regulating lipid metabolism [32]. Previously, L-FABP was found to be co-expressed with VEGF in the cell membrane [8], and other studies have suggested that lipid rafts are capable of acting in signaling platforms [33-35]. In a similar manner, our confocal microscopy images showed that L-FABP and VEGFR2 co-localized on the cell surface. Furthermore, levels of downstream signaling proteins, including Src/ FAK and PI3K/Akt, increased in the membrane fraction. Knockdown of L-FABP and VEGFR2 in Hus/L-FABP cells decreased the phosphorylation of these downstream signal molecules (Supplementary Figure 7D and 10). Further results also suggested that L-FABP was required for promotion of VEGFR2 activity (Supplementary Figure 11). Taken together, our findings provide evidence that L-FABP activates VEGFR2 signaling in HCC cells.

The regulation of VEGF in $\mathrm{HCC}$ has been highlighted because the related pathway plays an important role in cancer progression [2]. Indeed, only anti-VEGFR2 therapy has provided a significant benefit to clinical HCC patients and been approved by the FDA [4]. Here, we found that VEGF-A expression was regulated by the PI3K/ Akt pathway and HIF-1 $\alpha$, as the transcriptional activity of VEGF-A was significantly increased by L-FABP overexpression and reduced by deletion of the HIF- $1 \alpha$ binding site on the VEGF-A promoter. The higher level of secreted VEGF-A observed using the human growth factor array further supported our findings (Supplementary Figure 12). Interestingly, a previous study also showed that L-FABP levels were positively correlated with levels of VEGF-A mRNA [7]. When considered together, these results suggest that L-FABP may regulate VEGF-A 
expression in $\mathrm{HCC}$ cells via the PI3K/Akt pathway in a HIF- $1 \alpha$-dependent manner.

In previous studies, L-FABP-knockout mice showed decreased lipid metabolism and increased incidence of the obese phenotype with a high-fat diet $[32,36]$. Mutation studies revealed that the binding of L-FABP to phospholipids was significantly decreased in the Phe3-toTrp mutant, and Lys31 contributed to phospholipid binding $[22,23]$. Other studies have also suggested that the T94A mutation alters the structure and stability of L-FABP and causes a loss of function [24-26]. In our experiment, F3W, $\mathrm{K} 31 \mathrm{E}$, and T94A mutations exhibited decreased levels of VEGF and reduced angiogenic potential; however, reduced migration activity was observed in $\mathrm{K} 31 \mathrm{E}$ and T94A only. The effects of a membrane cholesterol depletion agent (MBCD) [37] and cholesterol-lowering compounds (filipin and simvastatin) [38] (Supplementary Figure 13) further supported the findings of the mutation investigation, with results suggesting that the cholesterolassociating activity of L-FABP was important for its function. The T94A mutation of L-FABP further disrupts the interaction of L-FABP and VEGFR2 (Supplementary Figure 14). Although the precise mechanisms still need further investigation, these findings indicate that the function of L-FABP in the cell membrane is related not only to metabolism but also to oncogenic potential.

$\mathrm{HCC}$ is a highly heterogeneous disease displaying differences in angiogenesis, extracellular matrix proteins, and tumor cell microenvironment [39]. Because VEGF levels are correlated with HCC malignancy and poor prognosis [40], previous studies focused on angiogenic heterogeneity, especially the relationship of VEGF and HIF-1 $\alpha$ expression to HCC [4]. Whether or not L-FABP and VEGF-A, in addition to other onco-proteins, also show correlated expression in benign hepatocyte cell lines (e.g., Hus and Chang) or HCC cell lines is an interesting question, but little information is currently available. Furthermore, the copy number or methylation status of genes of interest should also be analyzed in future studies. A recent study revealed that L-FABP promotes diet-induced fatty liver disease and hepatic steatosis [41], but L-FABP expression was not significantly correlated with clinical pathologic characteristics (including age, sex, grade, invasion, metastasis, and stage) in the present study (Table 2). However, we found that L-FABP was significantly up-regulated in HCC patients with and without cirrhosis. Moreover, in the cirrhosis patients, high L-FABP expression was related to a higher risk of poor survival (Supplementary, Figure 15). Previous studies suggested that the "angiogenic switch" is required for the formation of a solid HCC tumor [42], and that VEGF is involved in an autocrine feed-forward loop that triggers angiogenesis $[43,44]$. Because the relationship between L-FABP expression and HCC progression remains unclear, and because there is no appropriate prognosis marker for
HCC with cirrhosis [45], L-FABP may serve as a potential research target in further studies.

In summary, our study revealed for the first time that L-FABP potently induces up-regulation of VEGF-A and increases angiogenic potential and migration activity in HCC cells. Our results also suggest that the function of L-FABP in HCC could be influenced by mutations in its cholesterol interaction sites. Together with previous reports, our findings indicate that L-FABP is a potential therapeutic target in $\mathrm{HCC}$ therapy.

\section{MATERIALS AND METHODS}

\section{Subjects and tissues}

Tumor and normal adjacent tissue (NAT) from 90 HCC patients were purchased from US Biomax, Inc. (array number: HLiv-HCC180Sur-02); the patients included 12 females and 78 males, with an average age of $53.5 \pm 10.0$ years (Table 3 ).

\section{Antibodies and chemical inhibitors}

Antibodies specific to L-FABP, VEGF-A, flotillin-2, lamin $\mathrm{A} / \mathrm{C}, \alpha$-tubulin, and $\beta$-actin were purchased from Santa Cruz Biotechnology (Texas, USA). Antibodies specific to VEGFR2, phospho-VEGFR2, protooncogene tyrosine-protein kinase $\mathrm{Src}(\mathrm{Src})$, phosphoSrc, focal adhesion kinase 1 (FAK), phospho-FAK, phosphatidylinositol 3-kinase regulatory subunit (p85), RAC-alpha serine/threonine-protein kinase (Akt), phospho-Akt, serine/threonine-protein kinase mTOR (mTOR), phospho-mTOR, eukaryotic translation initiation factor 4E-binding protein 1 (4EBP1), phospho4EBP1, and HIF-1 $\alpha$ were obtained from Cell Signaling Technology. The chemical inhibitors Src inhibitor I and sorafenib were obtained from Calbiochem (Darmstadt, Germany) and Selleckchem (Boston, USA), respectively.

\section{Tissue microarray construction and immunohistochemistry}

The tissue array sections were immunostained with specific antibodies against L-FABP (1:100) and VEGF-A (1:100). Pathologists at GenDiscovery Biotechnology, Inc. (Taipei City, Taiwan) interpreted the staining results, which were analyzed for the intensity and percentage of staining area by using Quick-score analysis, whereby scores $(\mathrm{Q})$ were calculated as follows: $\mathrm{Q}=$ Percentage of positive cells $(\mathrm{P}) \times$ Intensity $(\mathrm{I})$; maximum $\mathrm{Q}=300$. The results were then graded according to the following criteria: 1: $\mathrm{Q}=0-99$, weak staining; $2: \mathrm{Q}=100-199$, moderate staining; 3: $Q=200-299$, strong staining; and $4: \mathrm{Q}=300$, very strong staining. 
Table 2: Association of L-FABP protein expression with clinical pathologic characteristics in patients with HCC

\begin{tabular}{|c|c|c|c|c|}
\hline Characteristics & Low (1) & Intermediate (2) & High (3) & $P$ value \\
\hline Age (years) Mean \pm SD & $53.2 \pm 8.5$ & $52.4 \pm 10.6$ & $59.3 \pm 8.2$ & $0.089^{\mathrm{a}}$ \\
\hline Age $>=53.5$ & $9(47.4)$ & $30(51.7)$ & $8(66.7)$ & $0.555^{\mathrm{b}}$ \\
\hline \multicolumn{5}{|l|}{ Sex } \\
\hline Female & $4(21.1)$ & $7(11.9)$ & $1(8.3)$ & $0.509^{b}$ \\
\hline Male & $15(78.9)$ & $52(88.1)$ & $11(91.7)$ & \\
\hline Grade & & & & $0.484^{b}$ \\
\hline G1 & $2(10.5)$ & $3(5.1)$ & $1(8.3)$ & \\
\hline G2 & $15(78.5)$ & $46(78.0)$ & $7(58.3)$ & \\
\hline G3 & $2(10.5)$ & $10(16.9)$ & $4(33.3)$ & \\
\hline pT (invasion depth) & & & & $0.169^{b}$ \\
\hline $\mathrm{T} 1$ & $2(10.5)$ & $8(14.3)$ & $2(16.7)$ & \\
\hline $\mathrm{T} 2$ & $9(47.4)$ & $20(35.7)$ & $2(16.7)$ & \\
\hline $\mathrm{T} 3$ & $6(31.6)$ & $28(50.0)$ & $7(58.3)$ & \\
\hline $\mathrm{T} 4$ & $2(10.5)$ & $0(0.0)$ & $1(8.3)$ & \\
\hline pN (lymph node metastasis) & & & & $0.759^{\mathrm{b}}$ \\
\hline No & $19(100.0)$ & $54(98.2)$ & $11(100.0)$ & \\
\hline N1 & $0(0.0)$ & $1(1.8)$ & $0(0.0)$ & \\
\hline pM (distant metastasis) & & & & $0.578^{b}$ \\
\hline M0 & $19(100.0)$ & $54(96.4)$ & $11(100.0)$ & \\
\hline M1 & $0(0.0)$ & $2(3.6)$ & $0(0.0)$ & \\
\hline TNM stage & & & & $0.546^{\mathrm{b}}$ \\
\hline I & $2(10.5)$ & $8(14.3)$ & $2(16.7)$ & \\
\hline II & $9(47.4)$ & $20(35.7)$ & $2(16.7)$ & \\
\hline III & $8(42.1)$ & $25(44.6)$ & $8(66.7)$ & \\
\hline IV & $0(0.0)$ & $3(5.4)$ & $0(0.0)$ & \\
\hline
\end{tabular}

Abbreviations: HCC, hepatocellular carcinoma; N, number.

a. ANOVA test.

b. Chi-square test.

Table 3: Clinical characteristics of the cases included in analyses of L-FABP protein expression evaluated by immunohistochemistry

\begin{tabular}{lcccc}
\hline Characteristics & NAT, $N=\mathbf{9 0}$ & $\begin{array}{c}\text { HCC without } \\
\text { cirrhosis, } N=\mathbf{5 7}\end{array}$ & $\begin{array}{c}\text { HCC with cirrhosis, } \\
N=\mathbf{3 3}\end{array}$ & P value \\
\hline Age (years), Mean \pm SD & $53.5 \pm 10.0$ & $54.2 \pm 10.3$ & $52.2 \pm 9.6$ & $0.650^{\text {a }}$ \\
Sex, N (\%) & $8(13.3)$ & $6(10.5)$ & $6(18.2)$ & $0.589^{\mathrm{b}}$ \\
Female & $78(86.7)$ & $51(89.5)$ & $27(81.8)$ & \\
Male &
\end{tabular}

Abbreviations: HCC, hepatocellular carcinoma; N, number.
a. ANOVA test.
b. Chi-square test.
c. One age miss data. 


\section{Cell culture}

Huh7 and HepG2 cells were obtained from the Japanese Collection of Research Bioresources (National Institute of Health Sciences, Japan) and maintained in Dulbecco's modified Eagle's medium (DMEM) with 10\% FBS. The immortalized cell line, which was derived from human primary hepatocytes, Hus-E/2 (Hus cells), was cultured in primary hepatocyte $(\mathrm{PH})$ medium (DMEM containing $20 \mathrm{mM}$ HEPES, $15 \mu \mathrm{g} / \mathrm{ml}$ L-proline, 0.25 $\mu \mathrm{g} / \mathrm{ml}$ insulin, $50 \mathrm{nM}$ dexamethasone, $44 \mathrm{mM}$ sodium bicarbonate, $10 \mathrm{mM}$ nicotinamide, $5 \mathrm{ng} / \mathrm{ml} \mathrm{EGF}$, and 0.1 $\mathrm{mM}$ ascorbic acid) with $10 \%$ FBS. All cell lines were incubated in a $5 \% \mathrm{CO} 2$ atmosphere at $37^{\circ} \mathrm{C}$.

\section{Creation and culture of L-FABP-overexpressed stable clones}

To construct pcDNA3.1/L-FABP, a full-length L-FABP cDNA fragment (1-121 aa), which was cloned from the cDNA of Huh7 cells, was inserted into the pcDNA3.1 vector via the TOPO PCR cloning system (Life Technologies, NY, USA). The construct was validated using nucleotide sequencing. Hus cells were transfected with pcDNA3.1/L-FABP using Lipofectamine 2000 reagent (Invitrogen, NY, USA). After 2-4 weeks of culture in a medium containing $1 \mathrm{mg} / \mathrm{ml} \mathrm{G} 418$ (SigmaAldrich, USA), stable clones were selected. Each clone was analyzed for L-FABP expression twice per month using western blots.

\section{Western blot analysis and immunoprecipitation}

Proteins $(50 \mu \mathrm{g})$ were resolved by $10 \%$ sodium dodecyl sulfate (SDS)-polyacrylamide gel electrophoresis and transferred to polyvinylidene fluoride membranes (Millipore, MA, USA). The membrane was first incubated with primary antibodies followed by horseradish peroxidase-conjugated secondary antibodies (Chemicon International). Signals were visualized using enhanced chemiluminescence detection reagent from Millipore, and the images were obtained using a Luminescence/ Fluorescence Imaging System (LAS-4000; Fuji).

For immunoprecipitation, cell lysates containing 500 $\mu \mathrm{g}$ of protein were pre-cleared by protein A/G Sepharose beads (Millipore) and then incubated with anti-L-FABP or anti-VEGFR2 antibody overnight at $4{ }^{\circ} \mathrm{C}$. The immune complexes were washed three times in ice-cold PBS and subsequently captured by protein A/G Sepharose beads, and then the immunoprecipitated proteins were subjected to western blot analysis.

\section{Cell migration assay}

To study 2D migration activity, cells were seeded on a $35-\mathrm{mm}$ cell culture $\mu$-Dish (ibidi, Planegg, Germany) at a density of $4 \times 10^{5}$ cells per $\mathrm{cm}^{2}$ for a wound healing assay. Two days after seeding, the insert was removed with tweezers, yielding a standardized wound of $500 \mu \mathrm{m}$. The dish was washed with PBS and subsequently imaged for 0,6 , and $24 \mathrm{~h}$.

To study 3D migration activity, cells were maintained in serum-free medium for $24 \mathrm{~h}$ and then seeded into Transwell Boyden chambers (Millipore). Subsequently, they were incubated in complete medium with $10 \%$ fetal bovine serum at $37^{\circ} \mathrm{C}$ for $16 \mathrm{~h}$. The cells on the bottom side of the membrane were fixed with $1 \%$ formaldehyde/PBS for $15 \mathrm{~min}$, stained with $0.1 \%$ crystal violet for $40 \mathrm{~min}$, and then counted using an inverted contrast light microscope (Olympus CKX-41 with NIKON DSU-3 digital sight image system).

\section{Angiogenesis activity assay}

\section{Cell culture}

Primary human umbilical vein endothelial cells (HUVECs) (Sciencell, CA, USA) were grown in M199 medium (containing $100 \mu \mathrm{g} / \mathrm{ml}$ Endothelial Cell Growth Supplement, $10 \mathrm{ng} / \mathrm{ml}$ heparin, and $5 \%$ fetal bovine serum) and cultured in a $5 \% \mathrm{CO} 2$ atmosphere at $37^{\circ} \mathrm{C}$.

\section{In vitro tube formation assay}

A 24-well plate was coated with $100 \mu$ of Matrigel (1 $\mathrm{mg} / \mathrm{ml}$; BD Biosciences, CA, USA), which was allowed to solidify at $37^{\circ} \mathrm{C}$ for $1 \mathrm{~h}$. HUVECs $\left(1 \times 10^{4}\right.$ cells/well) were seeded on the Matrigel and incubated with conditioned medium collected from cultured cells (Huh7 cells: L-FABP-overexpressing or L-FABPstable knockdown) for 8-12 h. The VEGF group was used to check the angiogenic activity of HUVEC cells. Photographs from random fields were acquired using a DP-50 microscope (Olympus, Tokyo, Japan), and each image was quantified according to the following formula [46]: Angiogenic score $=[($ No. of sprouting cells $) \times 1+$ (No. of connected cells $) \times 2+($ No. of polygons $) \times 3)] /$ Total number of cells $+[0,1$, or 2]. The definition of cell types and the parameters 0,1 , and 2 can be found in previous studies [9]. In brief, the presence of a complex mesh (luminal structures consisting of walls with a thickness of two to three cells) was given a score of 1 . If this complex structure was present and the walls had a thickness of four or more cells thick, then a score of 2 was given. The absence of a complex mesh resulted in a score of 0 points.

\section{In vivo Matrigel plug assay}

Matrigel (phenol red-free; BD Biosciences) was mixed with L-FABP stable clones $\left(2 \times 10^{6}\right.$ cells/Matrigel $)$. The Matrigel plugs were subcutaneously injected into 10 4-week-old male NOD/SCID mice (one per mouse; 
see Animal Experiments below for details of mice) and recovered on Day 10 for analysis.

\section{Short interference RNA (siRNA) and short hairpin RNA (shRNA)}

Modified oligonucleotides used as siRNA for L-FABP and control siRNA were obtained from Invitrogen. The shRNA clones were purchased from the National RNAi Core Facility Platform, Taiwan. For transfection, $1 \times 10^{5} \mathrm{Hus} / \mathrm{L}-\mathrm{FABP}$ or Huh7 cells were plated in a six-well plate for $24 \mathrm{~h}$, and Lipofectamine 2000 was used to transfect siRNA or shRNA for knockdown of protein expression [47].

\section{Purification of L-FABP recombinant protein}

L-FABP recombinant protein was purified using the Ni-NTA Purification system (Novex, USA) as previously described [48]. In particular, L-FABP-overexpressing Hus cells (transfected with the pcDNA3.1D/L-FABP plasmid) were lysed and sonicated in a native condition. After centrifugation, the supernatant was transferred to a fresh tube and allowed to slowly flow through the Ni-NTA resin by gravity. Subsequently, the resin was washed five times using Native Wash buffer (with 20 $\mathrm{mM}$ imidazole), eluted in $1 \mathrm{~mL}$ of Native Elution buffer (with $250 \mathrm{mM}$ imidazole), and analyzed using SDS-PAGE (Supplementary Figure 4).

\section{Overlay assay (far-western blot)}

The interaction between L-FABP and VEGFR2 (intracellular domain) was evaluated using the overlay assay as previous described [49]. To estimate the affinity of L-FABP and VEGFR2, an increased amount of VEGFR2 purified protein (\#P3871, Abnova, USA) was loaded on SDS-PAGE and transferred to a PVDF membrane. After blocking, the membrane was washed and overlaid with V5-tagged L-FABP recombinant protein $(1 \mu \mathrm{g} / \mathrm{ml})$ prepared by the methods described above for 12 hours, followed by blotting with an anti-V5 antibody (Invitrogen) and HRP-conjugated secondary antibody. The signals were detected using enhanced chemiluminescence (ECL; Millipore) and recorded using a luminescence imaging system. Results were quantified by ImageJ software, and the affinity constant (Kd) of the interaction was determined by the non-linear regression fitting function of the GraphPad Prism 5 program (GraphPad Software).

\section{Lipid raft isolation}

Raft microdomains were purified using a previously described method [50]. Briefly, $700 \mu \mathrm{l}$ of $1 \%$ Triton $\mathrm{X}-100$ lysis buffer was applied to pre-washed cells, and a Teflon-coated dounce homogenizer was used to disrupt the cell membranes (20-30 strokes). The lysate
(4 mg) was incubated at $4{ }^{\circ} \mathrm{C}$ for $30 \mathrm{~min}$ and mixed with an equivalent volume of $80 \%$ sucrose solution to yield a $40 \%$ sucrose gradient, and the mixture was transferred to a $12-\mathrm{ml}$ polyallomer ultracentrifuge tube (suitable for an SW41 rotor) (Beckman Instruments). Subsequently, 6.5 and $3.5 \mathrm{ml}$ of $30 \%$ and $5 \%$ sucrose cushion, respectively, was overlaid on the sample, and ultracentrifugation was applied at $187,813 \mathrm{~g}$ and $4^{\circ} \mathrm{C}$ for $20 \mathrm{~h}$ using an SW41 rotor. The floating opaque band corresponding to the detergent-resistant lipid rafts was collected and subjected to western blot analysis.

\section{Confocal microscopy analysis}

L-FABP-stably expressing Hus cells were seeded onto a $22 \times 22 \mathrm{~mm}$ cover slide, washed, fixed, and then permeabilized with $0.25 \%$ Triton X-100 for $10 \mathrm{~min}$. For double-staining, the slides were first incubated with L-FABP and VEGFR2 primary antibodies overnight, then stained with Alexa488 (anti-mouse) and Alexa568 (anti-rabbit) $(20 \mathrm{mU} / \mathrm{mL})$ for $1 \mathrm{~h}$ in darkness, and finally counter-stained for nuclei with DAPI $(10 \mathrm{ng} / \mathrm{mL})$ for $10 \mathrm{~min}$. The images were captured and analyzed using a Leica TCS SP5 Spectral Confocal Microscope (Leica Microsystems).

\section{Small GTPase binding assay}

Cells $\left(1 \times 10^{7}\right)$ were seeded and collected in $0.4 \mathrm{ml}$ of ice-cold lysis buffer (50 mM Tris-HCl, $\mathrm{pH} 7.5,10 \mathrm{mM}$ $\mathrm{MgCl} 2,500 \mathrm{mM} \mathrm{NaCl}, 1 \%$ Triton $\mathrm{X}-100$, and protease inhibitor cocktail). After lysing for $20 \mathrm{~min}$ on ice, cell debris was removed by centrifugation at $300 \mathrm{~g}$ and $4{ }^{\circ} \mathrm{C}$ for $10 \mathrm{~min}$. Half of each lysate (i.e., $50 \mu \mathrm{g}$ of protein) was mixed with $15 \mu$ of GST-PBD or GST-RBD beads, as recommended by a previous study [51], and incubated for $1 \mathrm{~h}$ at $4^{\circ} \mathrm{C}$ with rotation. In preparation for western blot analysis, samples were centrifuged $(3,000 \mathrm{~g}$ for $1 \mathrm{~min}$ at $\left.4{ }^{\circ} \mathrm{C}\right)$, washed twice in ice-cold wash buffer $(25 \mathrm{mM}$ Tris- $\mathrm{HCl}, \mathrm{pH} 7.5,30 \mathrm{mM} \mathrm{MgCl}$, and $40 \mathrm{mM} \mathrm{NaCl}$ ), and finally resuspended in $30 \mu \mathrm{l}$ of SDS sample buffer and heated at $100^{\circ} \mathrm{C}$ for $5 \mathrm{~min}$.

\section{Construction of human VEGF-A promoter}

The VEGF-A promoter (full-length 1190 bp: from -1127 to +73 ) was synthesized by ShineGene Molecular Biotech Inc. (Shanghai, China) and constructed into the puc57 vector. Cutting of the full-length promoter with SacI and HindIII restriction enzymes allowed it to be cloned into the pGL4.22 luciferase reporter vector. The generated 5' serial deletion constructs of the VEGF-A promoter, representing deletion of the HIF-1 $\alpha$ binding site (the major transcription factor for regulation of VEGF-A expression), were named as follows: D1: bp -901 to +73 ; D2: bp -782 to +73 ; D3: bp -199 to +73 . The primers used in the aforementioned cloning are listed in Supplementary 
Table 1. Nucleotide sequencing was used to validate all constructs.

\section{Luciferase reporter assay}

L-FABP-overexpressing Hus cells were transfected with the constructed pGL4.22/VEGF-A promoter plasmids and the pGL4-Renilla luciferase control reporter plasmid as an internal control. After transfection with Lipofectamine 2000 and $24 \mathrm{~h}$ of incubation, the cells were lysed and luciferase activity was determined using the Dual-Luciferase Reporter Assay System (Promega) according to the manufacturer's protocol, in addition to a SpectraMax L luminometer (Molecular Devices, CA, USA).

\section{Chromatin immunoprecipitation assay}

The chromatin immunoprecipitation assay was performed as follows. The cell lysate of Hus/L-FABP or control cells was sonicated, and then the chromatin was immunoprecipitated with HIF-1 $\alpha$ antibody or rabbit immunoglobulin G antibody (Santa Cruz Biotechnology) as a negative control. After precipitation, the bound DNA was dissolved with $40 \mu \mathrm{l}$ of ddH2O and then amplified by PCR with primers amplifying the HIF-1 $\alpha$ binding element (-1041 to -750 , Supplementary Table 1). The final PCR products were analyzed using $1.8 \%$ agarose gels and visualized by ethidium bromide staining.

\section{Animal experiments}

All animal experiments were conducted according to regulations approved by the Institutional Animal Care and Use Committee of College of Medicine, National Taiwan University. Male NOD-SCID mice (4 weeks old) were obtained from LASCO Taiwan Co., Ltd. For xenograft experiments, and Hus/L-FABP or Hus/Vector cells $(2 \times$ $10^{6}$ cells for each) were suspended in $200 \mu \mathrm{l}$ of OPTIMEM (Invitrogen) and inoculated into the right hindlimb of each mouse. Tumor size was measured twice per week with calipers, and the tumor volume was estimated using the following formula: (width) ${ }^{2} \times$ length $/ 2$, as described in previous studies [52]. After 8 weeks, the mice were sacrificed and the tumors were removed, measured, and processed for immunohistochemistry.

To conduct a metastasis assay, we used a lung metastasis model described in previous studies [53]. Specifically, Hus/L-FABP or Hus/Vector cells $\left(4 \times 10^{6}\right.$ cells each) were suspended in $100 \mu \mathrm{l}$ of OPTI-MEM and introduced intravenously into the tail vein of male NOD/ SCID mice. The mice were sacrificed after 10 weeks. Metastatic colonies in the lungs of mice were counted and photographed, and the removed lungs were fixed and embedded in paraffin for immunohistochemical analysis.

\section{Cloning of L-FABP mutants}

The amino acid substitution of wild-type L-FABP protein was conducted as follows. L-FABP pointmutation clones, including Phe3 to Trp (F3W), Lys31 to Glu (K31E), and Thr94 to Ala (T94A), were generated using the QuickChange Site-Directed Mutagenesis Kit (Stratagene). The primers for PCR reactions and subsequent treatments with DpnI to eliminate the template DNA are listed in Supplementary Table 1. All constructs were validated using nucleotide sequencing.

\section{Statistical analysis}

Relationships between protein expression and categorical variables were analyzed using chi-squared tests. For multivariate analysis, independent prognostic factors were determined using Cox's proportional hazard model. Survival curves were calculated using the KaplanMeier method and compared using log-rank tests. In vitro and in vivo experiments were analyzed in GraphPad Prism 5 (GraphPad Software), with the data presented as the mean \pm standard error of the mean (SEM). Statistical significance was defined as $p<0.05$.

\section{ACKNOWLEDGMENTS}

We thank Dr. Huang Cheng from the Ming-Fu Chang Laboratory, Biochemistry and Molecular Biology, College of Medicine, National Taiwan University, for donating the human immortalized hepatocyte (Hus) cells.

\section{FUNDING}

This work was supported by grants from the Ministry of Science and Technology of the Republic of China (MOST103-2320-B-003-001-MY3). The funder had no role in study design, data collection and analysis, or decision to publish the manuscript.

\section{CONFLICTS OF INTEREST}

No conflict of interest exists in the submission of this manuscript, and the manuscript was approved by all authors for publication.

\section{REFERENCES}

1. Kaseb AO, Hanbali A, Cotant M, Hassan MM, Wollner I, Philip PA. Vascular endothelial growth factor in the management of hepatocellular carcinoma: a review of literature. Cancer. 2009; 115:4895-4906.

2. Moeini A, Cornellà H, Villanueva A. Emerging signaling pathways in hepatocellular carcinoma. Liver Cancer. 2012; 1:83-93. 
3. Romanque P, Piguet AC, Dufour JF. Targeting vessels to treat hepatocellular carcinoma. Clin Sci (Lond). 2008; 114:467-477.

4. Zhu AX, Duda DG, Sahani DV, Jain RK. HCC and angiogenesis: possible targets and future directions. Nat Rev Clin Oncol. 2011; 8:292-301.

5. Storch J, McDermott L. Structural and functional analysis of fatty acid-binding proteins. J Lipid Res. 2009; 50:S126-131.

6. Woodford JK, Behnke WD, Schroeder F. Liver fatty acid binding protein enhances sterol transfer by membrane interaction. Mol Cell Biochem. 1995; 152:51-56.

7. Dong LH, Li H, Wang F, Li FQ, Zhou HY, Yang HJ. Expression of liver-type fatty acid-binding protein and vascular endothelial growth factor and their correlation in human hepatocellular carcinoma. Nan Fang Yi Ke Da Xue Xue Bao. 2007; 27:318-321.

8. Li J, Dong L, Wei D, Wang X, Zhang S, Li H. Fatty acid synthase mediates the epithelial-mesenchymal transition of breast cancer cells. Int J Biol Sci. 2014; 10:171-180.

9. Nevo J, Mai A, Tuomi S, Pellinen T, Pentikäinen OT, Heikkilä P, Lundin J, Joensuu H, Bono P, Ivaska J. Mammary-derived growth inhibitor (MDGI) interacts with integrin $\alpha$-subunits and suppresses integrin activity and invasion. Oncogene. 2010; 29:6452-6463.

10. Takeuchi Y, Fukunaga K. Differential subcellular localization of two dopamine D2 receptor isoforms in transfected NG108-15 cells. J Neurochem. 2003; 85:1064-1074.

11. Shioda N, Takeuchi Y, Fukunaga K. Advanced research on dopamine signaling to develop drugs for the treatment of mental disorders: proteins interacting with the third cytoplasmic loop of dopamine D2 and D3 receptors. J Pharmacol Sci. 2010; 114:25-31.

12. Storch J, Thumser AE. Tissue-specific functions in the fatty acid-binding protein family. J Biol Chem. 2010; 285:32679-32683.

13. McIntosh AL, Atshaves BP, Storey SM, Landrock KK, Landrock D, Martin GG, Kier AB, Schroeder F. Loss of liver FA binding protein significantly alters hepatocyte plasma membrane microdomains. J Lipid Res. 2012; 53:467-480.

14. Rousseau S, Houle F, Kotanides H, Witte L, Waltenberger J, Landry J, Huot J. Vascular endothelial growth factor (VEGF)-driven actin-based motility is mediated by VEGFR2 and requires concerted activation of stressactivated protein kinase 2 (SAPK2/p38) and geldanamycinsensitive phosphorylation of focal adhesion kinase. J Biol Chem. 2000; 275:10661-10672.

15. Koch S, Tugues S, Li X, Gualandi L, Claesson-Welsh L. Signal transduction by vascular endothelial growth factor receptors. Biochem J. 2011; 437:169-183.

16. Claesson-Welsh L, Welsh M. VEGFA and tumour angiogenesis. J Intern Med. 2013; 273:114-127.
17. Karar J, Maity A. PI3K/AKT/mTOR Pathway in Angiogenesis. Front Mol Neurosci. 2011; 4:51.

18. Skinner HD, Zheng JZ, Fang J, Agani F, Jiang BH. Vascular endothelial growth factor transcriptional activation is mediated by hypoxia-inducible factor 1alpha, HDM2, and p70S6K1 in response to phosphatidylinositol 3-kinase/AKT signaling. J Biol Chem. 2004; 279:45643-45651.

19. Park MS, Kim BR, Dong SM, Lee SH, Kim D, Rho SB. The antihypertension drug doxazosin inhibits tumor growth and angiogenesis by decreasing VEGFR-2/Akt/mTOR signaling and VEGF and HIF-1 $\alpha$ expression. Oncotarget. 2014; 5:4935-4944. doi: 10.18632/oncotarget.2064.

20. Dodd KM, Yang J, Shen MH, Sampson JR, Tee AR. mTORC1 drives HIF-1 $\alpha$ and VEGF-A signalling via multiple mechanisms involving 4E-BP1, S6K1 and STAT3. Oncogene. 2015; 34:2239-2250.

21. Segrelles C, Ruiz S, Santos M, Martínez-Palacio J, Lara MF, Paramio JM. Akt mediates an angiogenic switch in transformed keratinocytes. Carcinogenesis. 2004; 25:1137-1147.

22. Davies JK, Thumser AEA, Wilton DC. Binding of recombinant rat liver fatty acid-binding protein to small anionic phospholipid vesicles results in ligand release: a model for interfacial binding and fatty acid targeting. Biochemistry. 1999; 38:16932-16940.

23. Davies JK, Hagan RM, Wilton DC. Effect of charge reversal mutations on the ligand- and membrane-binding properties of liver fatty acid-binding protein. J Biol Chem. 2002; 277:48395-48402.

24. Huang $\mathrm{H}$, McIntosh AL, Martin GG, Landrock KK, Landrock D, Gupta S, Atshaves BP, Kier AB, Schroeder F. Structural and functional interaction of fatty acids with human liver fatty acid-binding protein (L-FABP) T94A variant. FEBS J. 2014; 281:2266-2283.

25. Martin GG, McIntosh AL, Huang H, Gupta S, Atshaves BP, Landrock KK, Landrock D, Kier AB, Schroeder F. The human liver fatty acid binding protein T94A variant alters the structure, stability, and interaction with fibrates. Biochemistry. 2013; 52:9347-9357.

26. Gao N, Qu X, Yan J, Huang Q, Yuan HY, Ouyang DS. L-FABP T94A decreased fatty acid uptake and altered hepatic triglyceride and cholesterol accumulation in Chang liver cells stably transfected with L-FABP. Mol Cell Biochem. 2010; 345:207-214.

27. Wang G, Gong Y, Anderson J, Sun D, Minuk G, Roberts MS, Burczynski FJ. Antioxidative function of L-FABP in L-FABP stably transfected Chang liver cells. Hepatology. 2005; 42:871-879.

28. Yan J, Gong Y, She YM, Wang G, Roberts MS, Burczynski FJ. Molecular mechanism of recombinant liver fatty acid binding protein's antioxidant activity. J Lipid Res. 2009; 50:2445-2454.

29. Elmasri H, Ghelfi E, Yu Cw, Traphagen S, Cernadas M, Cao H, Shi GP, Plutzky J, Sahin M, Hotamisligil G, 
Cataltepe S. Endothelial cell-fatty acid binding protein 4 promotes angiogenesis: role of stem cell factor/c-kit pathway. Angiogenesis. 2012; 15:457-468.

30. Zhang L, Wang JN, Tang JM, Kong X, Yang JY, Zheng F, Guo LY, Huang YZ, Zhang L, Tian L, Cao SF, Tuo CH, Guo HL, Chen SY. VEGF is essential for the growth and migration of human hepatocellular carcinoma cells. Mol Biol Rep. 2012; 39:5085-5093.

31. Leppänen V-M, Prota AE, Jeltsch M, Anisimov A, Kalkkinen N, Strandin T, Lankinen H, Goldman A, Ballmer-Hofer K, Alitalo K. Structural determinants of growth factor binding and specificity by VEGF receptor 2 . Proc Natl Acad Sci U S A. 2010; 107:2425-2430.

32. Furuhashi M, Hotamisligil GS. Fatty acid-binding proteins: role in metabolic diseases and potential as drug targets. Nat Rev Drug Discov. 2008; 7:489-503.

33. Simons K, Toomre D. Lipid rafts and signal transduction. Nat Rev Mol Cell Biol. 2000; 1:31-39.

34. Laurentiis Ad, Donovan L, Arcaro A. Lipid rafts and caveolae in signaling by growth factor receptors. Open Biochem J. 2007; 1:12-32.

35. Staubach S, Hanisch FG. Lipid rafts: signaling and sorting platforms of cells and their roles in cancer. Expert Rev Proteomics. 2011; 8:263-277.

36. Atshaves BP, Martin GG, Hostetler HA, McIntosh AL, Kier AB, Schroeder F. Liver fatty acid-binding protein and obesity. J Nutr Biochem. 2010; 21:1015-1032.

37. Cohen AW, Combs TP, Scherer PE, Lisanti MP. Role of caveolin and caveolae in insulin signaling and diabetes. Am J Physiol Endocrinol Metab. 2003; 285:E1151-1160.

38. Zhuang L, Kim J, Adam RM, Solomon KR, Freeman MR. Cholesterol targeting alters lipid raft composition and cell survival in prostate cancer cells and xenografts. J Clin Invest. 2005; 115:959-968.

39. Jeng KS, Chang CF, Jeng WJ, Sheen IS, Jeng CJ. Heterogeneity of hepatocellular carcinoma contributes to cancer progression. Crit Rev Oncol Hematol. 2015; 94:337-347.

40. Brodsky SV, Mendelev N, Melamed M, Ramaswamy G. Vascular density and VEGF expression in hepatic lesions. $\mathrm{J}$ Gastrointestin Liver Dis. 2007; 16:373-377.

41. Chen A, Tang Y, Davis V, Hsu FF, Kennedy SM, Song H, Turk J, Brunt EM, Newberry EP, Davidson NO. Liver fatty acid binding protein (L-Fabp) modulates murine stellate cell activation and diet-induced nonalcoholic fatty liver disease. Hepatology. 2013; 57:2202-2212.

42. Heindryckx F, Gerwins P. Targeting the tumor stroma in hepatocellular carcinoma. World J Hepatol 2015 Feb 27;7:165-76. 2015; 7:165-176.
43. Ferrara N. Vascular endothelial growth factor as a target for anticancer therapy. Oncologist. 2004; 9:2-10.

44. Chatterjee S, Heukamp LC, Siobal M, Schöttle J, Wieczorek C, Peifer M, Frasca D, Koker M, König K, Meder L, Rauh D, Buettner R, Wolf J, Brekken RA, Neumaier B, Christofori G, et al. Tumor VEGF:VEGFR2 autocrine feedforward loop triggers angiogenesis in lung cancer. J Clin Invest. 2013; 123:1732-1740.

45. Alkozai EM, Porte RJ, Adelmeijer J, Zanetto A, Simioni $\mathrm{P}$, Senzolo M, Lisman T. Levels of angiogenic proteins in plasma and platelets are not different between patients with hepatitis B/C-related cirrhosis and patients with cirrhosis and hepatocellular carcinoma. Platelets. 2014:1-6.

46. Aranda E, Owen GI. A semi-quantitative assay to screen for angiogenic compounds and compounds with angiogenic potential using the EA.hy926 endothelial cell line. Biol Res. 2009; 42:377-389.

47. Rosner M, Siegel N, Fuchs C, Slabina N, Dolznig H, Hengstschläger M. Efficient siRNA-mediated prolonged gene silencing in human amniotic fluid stem cells. Nat Protoc. 2010; 5:1081-1095.

48. Sun W, Lai Y, Li H, Nie T, Kuang Y, Tang X, Li K, Dunbar PR, Xu A, Li P, Wu D. High level expression and purification of active recombinant human interleukin-15 in Pichia pastoris. J Immunol Methods 2015 Dec 8 pii: S0022-1759(15)30067-3. 2015; S0022-1759:30067-30063.

49. Hall RA. Studying protein-protein interactions via blot overlay/far western blot. Methods Mol Biol. 2015; 1278:371-379.

50. Kim KB, Lee JS, Ko YG. The isolation of detergent resistant lipid rafts for two dimensional electrophoresis. Methods Mol Biol. 2008; 424:413-422.

51. Chang YC, Tien SC, Tien HF, Zhang H, Bokoch GM, Chang ZF. p210Bcr-Abl desensitizes Cdc42 GTPase signaling for SDF-1a-directed migration in chromic myeloid leukemia cells. Oncogene. 2009; 28:4105-4115.

52. Tao ZH, Wan JL, Zeng LY, Xie L, Sun HC, Qin LX, Wang L, Zhou J, Ren ZG, Li YX, Fan J, Wu WZ. miR-612 suppresses the invasive-metastatic cascade in hepatocellular carcinoma. J Exp Med. 2013; 210:789-803.

53. Tang SW, Yang TC, Lin WC, Chang WH, Wang CC, Lai MK, Lin JY. Nicotinamide N-methyltransferase induces cellular invasion through activating matrix metalloproteinase-2 expression in clear cell renal cell carcinoma cells. Carcinogenesis. 2011; 32:138-145. 